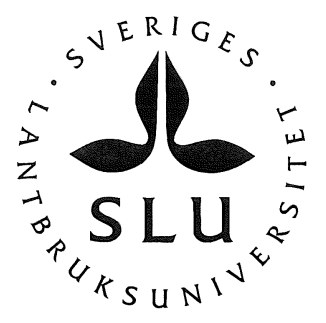

\title{
Nonparametric Estimation of the Variance of Sample Means Based on Nonstationary Spatial Data
}

\author{
Magnus Ekström
}

Arbetsrapport 882001

Working Paper 882001

SWEDISH UNIVERSITY OF

AGRICULTURAL SCIENCES (SLU)

Department of Forest Resource

Management and Geomatics

S-901 83 UMEA

Phone: 090-786 5825 Fax: 090-14 19 15, 778116
ISSN 1401-1204

ISRN SLU-SRG-AR--88 --SE 


\title{
NONPARAMETRIC ESTIMATION OF THE VARIANCE OF SAMPLE MEANS BASED ON NONSTATIONARY SPATIAL DATA
}

\author{
Magnus Ekström \\ Department of Forest Resource Management and Geomatics, \\ Swedish University of Agricultural Sciences \\ S-901 83 Umeå, Sweden
}

Keywords: Resampling; spatially indexed data; estimation of variance.

\begin{abstract}
In Politis and Romano (1993), different block resampling estimators of variance of general linear statistics, e.g. a sample mean, were proposed under the assumption of stationarity. In the present paper such estimators of variance of sample means, computed from nonstationary spatially indexed data $\left\{X_{i}\right.$ : $i \in \mathcal{A}\}$, where $\mathcal{A}$ is a finite subset of the integer lattice $\mathbb{Z}^{2}$, are studied. Consistency of estimators of variance will be shown for the following kind of data: Observations taken from different lattice points are allowed to come from different distributions, and the dependence structure is allowed to differ over the lattice. We assume that all observed values are from distributions with the same expected value, or with expected values that decompose additively into directional components. Furthermore, it will be assumed that observations separated by a certain distance are independent.
\end{abstract}

\section{INTRODUCTION}

Suppose we have spatially indexed data $\left\{X_{i}: i \in \mathcal{A}\right\}$, where $\mathcal{A}$ is a finite subset of the integer lattice $\mathbb{Z}^{2}$. Remote sensing data from satellites are, for example, on this form. Further, suppose that a statistic $s(\mathcal{A})$ is computed, which estimates some unknown parameter $\mu$, and that, in order to do inference, an estimate of the variance of $s(\mathcal{A})$ is desired.

For spatial lattice data, different block resampling estimators of variance have been proposed under the assumption of stationarity. See, for example, Possolo (1991), Politis and Romano (1993), Sherman and Carlstein (1994), and Sherman (1996). In the current paper such estimators of variance are studied in the case when the statistic in question is a sample mean computed 
from nonstationary spatial data. Such extentions are useful, since in many empirical applications, the hypothesis of stationarity can easily be rejected. The goal of this paper is to show that the resampling methods derived for dependent but stationary observations can still be employed (possibly in a slightly modified form) even if the assumption of stationarity is violated.

The kind of data we consider is of the following type: Observations from different points of the lattice are allowed to come from different distributions, but with the same expected value or with expected values that can be decomposed additively into directional components. Furthermore, the dependence structure is allowed to differ over the lattice, and observations separated by a certain "distance" will be assumed to be independent, as formalized in the following definition.

Definition 1 The r.v.s $X_{\boldsymbol{i}}, \boldsymbol{i}=\left(i_{1}, i_{2}\right) \in \mathcal{A}$, are said to be spatially $\boldsymbol{m}$ dependent if $X_{i^{\prime}}$ and $X_{i^{\prime \prime}}$ are independent whenever $\left|i_{1}^{\prime}-i_{1}^{\prime \prime}\right|>m_{1}$ or $\left|i_{2}^{\prime}-i_{2}^{\prime \prime}\right|>$ $m_{2}, \boldsymbol{m}=\left(m_{1}, m_{2}\right)$.

For simplicity, the case when $\mathcal{A}$ is rectangular will be considered, but extentions to non-rectangular subsets of $\mathbb{Z}^{2}$ (that possess some regularity) are possible. Henceforth we assume that $\mathcal{A}=\mathcal{A}_{n}=\left\{\boldsymbol{i}=\left(i_{1}, i_{2}\right): i_{1}=1, \ldots, n_{1}\right.$ and $i_{2}=$ $\left.1, \ldots, n_{2}\right\}$, where $\boldsymbol{n}=\left(n_{1}, n_{2}\right)$.

Remark. The results in this paper hold for more general data than indicated, i.e. the results are valid for arrays $\mathrm{X}_{n}=\left\{X_{i, n}: i \in \mathcal{A}_{n}\right\}$ of collections of r.v.s, such that for each $\boldsymbol{n}$, the r.v.s in $\mathrm{X}_{\boldsymbol{n}}$ are $\boldsymbol{m}$-dependent. To keep the notation manageable, we will write $X_{i}$ instead of $X_{i, n}$.

Define "rectangular" blocks of indices

$$
B_{\boldsymbol{i}}=\left\{\begin{array}{ccc}
\left(i_{1}, i_{2}\right) & \cdots & \left(i_{1}+k_{1}-1, i_{2}\right) \\
\vdots & \ddots & \vdots \\
\left(i_{1}, i_{2}+k_{2}-1\right) & \cdots & \left(i_{1}+k_{1}-1, i_{2}+k_{2}-1\right)
\end{array}\right\},
$$

and let $K=k_{1} k_{2}=\left|B_{\boldsymbol{i}}\right|$. Also, define $N=n_{1} n_{2}, X . .=\sum_{\boldsymbol{i} \in \mathcal{A}_{n}} X_{\boldsymbol{i}}$, and $\bar{X}=$ $X . . / N$. In the case when $E X_{\boldsymbol{i}}=\mu$, for all $\boldsymbol{i} \in \mathcal{A}_{\boldsymbol{n}}$, and all $\boldsymbol{n}$, the following estimators of $\gamma_{n}=\operatorname{Var}(\sqrt{N} \bar{X})$ will be considered:

Politis and Romano's (1993) jackknife estimator,

$$
\hat{\gamma}_{\boldsymbol{n}}^{(j e)}=\frac{K}{N^{\prime}} \sum_{i_{1}=1}^{n_{1}^{\prime}} \sum_{i_{2}=1}^{n_{2}^{\prime}}\left(\bar{X}_{B_{i}}-\bar{X}\right)^{2}
$$

where $\bar{X}_{B_{i}}=K^{-1} \sum_{j \in B_{i}} X_{j}, n_{i}^{\prime}=n_{i}-k_{i}+1, i=1,2$, and $N^{\prime}=n_{1}^{\prime} n_{2}^{\prime}$. 
Politis and Romano's (1993) bootstrap estimator,

$$
\hat{\gamma}_{n}^{(b e)}=\frac{K}{N^{\prime}} \sum_{i_{1}=1}^{n_{1}^{\prime}} \sum_{i_{2}=1}^{n_{2}^{\prime}}\left(\bar{X}_{B_{i}}-\bar{X}^{\prime}\right)^{2},
$$

where $\bar{X}^{\prime}=\left(N^{\prime}\right)^{-1} \sum_{i_{1}=1}^{n_{1}^{\prime}} \sum_{i_{2}=1}^{n_{2}^{\prime}} \bar{X}_{B_{i}}$. It should be noted that in the current setting, the bootstrap estimator above coincides with the estimator of variance suggested by Sherman (1996).

Politis and Romano's (1993) circular bootstrap estimator,

$$
\hat{\gamma}_{\boldsymbol{n}}^{(c b e)}=\frac{K}{N} \sum_{i \in \mathcal{A}_{n}}\left(\bar{X}_{B_{i}}-\bar{X}\right)^{2}
$$

where $X_{j}=X_{j^{\prime}}, j_{i}^{\prime}=j_{i}\left(\bmod n_{i}\right), i=1,2$, i.e., the data is wrapped around on a torus.

Next, let $X_{j}=\bar{X}$ for $\boldsymbol{j} \notin \mathcal{A}_{\boldsymbol{n}}$, and $\mathcal{A}_{\boldsymbol{n}}^{\prime}=\left\{\boldsymbol{i}: i_{1}=2-k_{1}, \ldots, n_{1}\right.$ and $i_{2}=$ $\left.2-k_{2}, \ldots, n_{2}\right\}$. Then there are $N^{\prime \prime}=\left(n_{1}+k_{1}+1\right)\left(n_{2}+k_{2}+1\right)$ different (overlapping) blocks $B_{\boldsymbol{i}}, \boldsymbol{i} \in \mathcal{A}_{\boldsymbol{n}}^{\prime}$, containing at least one $X_{j}, \boldsymbol{j} \in \mathcal{A}_{\boldsymbol{n}}$. By defining a bootstrap estimator of $\gamma_{\boldsymbol{n}}$ on these $N^{\prime \prime}$ blocks, we obtain an estimator which put more weight on the observations near the edges than what is the case with $\hat{\gamma}_{\boldsymbol{n}}^{(b e)}$ (or $\hat{\gamma}_{\boldsymbol{n}}^{(j e)}$ ). Further, such an estimator can be defined also for non-rectangular index sets (as described in, e.g., Sherman (1996)), which is an advantage over $\hat{\gamma}_{\boldsymbol{n}}^{(c b e)}$ which require the index set to be of rectangular shape. Thus, we suggest the following estimator of variance,

$$
\hat{\gamma}_{n}^{(n e)}=\frac{1}{K N} \sum_{i \in \mathcal{A}_{n}^{\prime}}\left(\sum_{j \in B_{i}}\left(X_{j}-\bar{X}\right) I_{j}\right)^{2},
$$

where $I_{j}$ is equal to 1 if $j \in \mathcal{A}_{n}$, and zero otherwise. Note that we use $N$ in the denominator rather than $N^{\prime \prime}$, since this gives an estimator with less bias (in the case of positively correlated r.v.s).

In Mercer and Hall (1911), yields of wheat on a $20 \times 25$ lattice of plots approximately 1 acre in total area were presented. Sherman (1996) estimated the variance of the sample mean of the Mercer and Hall data, using the bootstrap estimator $\hat{\gamma}_{\boldsymbol{n}}^{(b e)}$. It is clear, however, that this variance estimator (as well as $\hat{\gamma}_{\boldsymbol{n}}^{(j e)}, \hat{\gamma}_{\boldsymbol{n}}^{(c b e)}$, and $\hat{\gamma}_{\boldsymbol{n}}^{(n e)}$ ) can give erroneous results if the condition $E X_{\boldsymbol{i}}=\mu$, for all $\boldsymbol{i} \in \mathcal{I}_{\boldsymbol{n}}$, and all $\boldsymbol{n}$, is violated. According to Cressie (1993), Section 4.5, there is an irregular east-west trend in the mean structure of the wheat yield data, and the data should be detrended before an analysis requiring constant mean structure (or even more, stationarity) can be performed.

Assume we observe $Y_{\boldsymbol{i}}, \boldsymbol{i} \in \mathcal{A}_{\boldsymbol{n}}$, and that $\mu_{\boldsymbol{i}}=E Y_{\boldsymbol{i}}$ decomposes additively into directional components, as in the model for the wheat yield data proposed 
by Cressie. That is, $\mu_{i}=\mu+r_{i_{2}}+c_{i_{1}}$, where $\mu$ is the overall mean, $r_{i_{2}}$, $i_{2}=1, \ldots, n_{2}$, are the row effects, and $c_{i_{1}}, i_{1}=1, \ldots, n_{1}$, are the column effects. All effects, $\mu, r_{i_{2}}, i_{2}=1, \ldots, n_{2}$, and $c_{i_{1}}, i_{1}=1, \ldots, n_{1}$, may depend on $\boldsymbol{n}$. The effects can be estimated, and if, for example, ordinary least squares (OLS) is used, they can be estimated without any model or knowledge of the spatial dependency structure. Denote the OLS estimates by $\hat{\mu}, \hat{r}_{i_{2}}, i_{2}=1, \ldots, n_{2}$, and $\hat{c}_{i_{1}}, i_{1}=1, \ldots, n_{1}$. Estimate $\mu_{i}$ with $\hat{\mu}_{i}=\hat{\mu}+\hat{r}_{i_{2}}+\hat{c}_{i_{1}}$, and define residuals, $e_{i}=Y_{i}-\hat{\mu}_{i}, i \in \mathcal{A}_{n}$.

In this case we propose the following estimator of $\gamma_{\boldsymbol{n}}^{\prime}=\operatorname{Var}(\sqrt{N} \bar{Y})$,

$$
\hat{\gamma}_{\boldsymbol{n}}^{\left(n e^{\prime}\right)}=\frac{1}{K N} \sum_{\boldsymbol{i} \in \mathcal{A}_{n}^{\prime}}\left(\sum_{\boldsymbol{j} \in B_{\boldsymbol{i}}} e_{j} I_{j}\right)^{2},
$$

which, in fact, is the estimator $\hat{\gamma}_{n}^{(n e)}$ with the $X_{i}$-variables replaced by the residuals. The above estimators proposed by Politis and Romano, can all be modified in the same way to handle the situation when the mean value decomposes additively into directional components.

In the next section, assumptions under which the above estimators are consistent will be presented. In Section 3 there is a short discussion, and in Section 4 a simulation study is carried out in order to compare the different estimators. The proofs are given in Section 5.

\section{MAIN RESULTS}

We make the following assumptions.

AM: For all $\boldsymbol{n}, E X_{\boldsymbol{i}}=\mu, \boldsymbol{i} \in \mathcal{A}_{\boldsymbol{n}}$, where $\mu$ may depend on $\boldsymbol{n}$.

$\operatorname{AD}(\boldsymbol{m})$ : For all $\boldsymbol{n}$, the r.v.s $X_{\boldsymbol{i}}, \boldsymbol{i} \in \mathcal{A}_{\boldsymbol{n}}$, are spatially $\boldsymbol{m}$-dependent.

$\operatorname{AL}(\delta)$ : For some positive constants $\delta \leq 2$ and $\tau_{\delta}$, and for all $\boldsymbol{n}, E\left|X_{\boldsymbol{i}}\right|^{2+\delta}<$ $\tau_{\delta}<\infty, i \in \mathcal{A}_{n}$, i.e. we have uniformly bounded r.v.s.

$\operatorname{AK}(\delta)$ : If $\delta=2$, then $k_{i}=k_{i}\left(n_{i}\right)=o\left(n_{i}\right)$ as $k_{i}, n_{i} \rightarrow \infty, i=1,2$. If $0<$ $\delta<2$, then $\left(k_{1} / k_{2}\right)\left(\left(k_{1} k_{2} /\left(n_{1} n_{2}\right)\right) \log k_{2}\right)^{\delta},\left(k_{2} / k_{1}\right)\left(\left(k_{1} k_{2} /\left(n_{1} n_{2}\right)\right) \log k_{1}\right)^{\delta}$, and $\left(k_{i} / n_{i}\right) \log k_{i}, i=1,2$, all tend to zero as $k_{i}, n_{i} \rightarrow \infty, i=1,2$.

Theorem 1 If $\mathbf{A M}, \operatorname{AD}(\boldsymbol{m}), \operatorname{AL}(\delta)$, and $\mathbf{A K}(\delta)$ are valid, then

$$
\hat{\gamma}_{\boldsymbol{n}}^{(n e)}-\gamma_{\boldsymbol{n}} \stackrel{P}{\longrightarrow} 0 \text {, as } n_{1}, n_{2} \rightarrow \infty .
$$

Remark. It should be noted that in order to show consistency of the estimators (1)-(3) under the assumptions stated above, only slight modifications of the 
proof of Theorem 1 are needed.

The lengthy proof of Theorem 1 is given in Section 5. In the proof we will follow the same approach as in Belyaev (1996), where triangular arrays of rowwise $m$-dependent r.v.s were studied, and blockwise resampling schemes were proposed that consistently estimates the distribution of sums of r.v.s under assumptions similar to those in Theorem 1.

Next we consider the case when the considered r.v.s do not have a constant mean.

$\mathrm{AM}^{\prime}$ : For all $\boldsymbol{n}, Y_{\boldsymbol{i}}=X_{\boldsymbol{i}}+r_{i_{2}}+c_{i_{1}}$, where $X_{\boldsymbol{i}}, \boldsymbol{i} \in \mathcal{A}_{\boldsymbol{n}}$, satisfy assumption $\mathrm{AM}$, and $\sum_{i_{2}=1}^{n_{2}} r_{i_{2}}=0$ and $\sum_{i_{1}=1}^{n_{1}} c_{i_{1}}=0 . \mu, r_{i_{2}}$, and $c_{i_{1}}, \boldsymbol{i} \in \mathcal{A}_{\boldsymbol{n}}$, may all depend on $\boldsymbol{n}$.

The OLS estimators of the effects are

$$
\begin{aligned}
\hat{\mu} & =\bar{Y}=\frac{Y . .}{N}=\frac{1}{N} \sum_{i \in \mathcal{A}_{n}} Y_{i}, \\
\hat{r}_{i_{2}} & =\frac{Y_{i_{2}}}{n_{1}}-\hat{\mu}=\frac{1}{n_{1}} \sum_{i_{1}=1}^{n_{1}} Y_{i}-\hat{\mu}, \quad i_{2}=1, \ldots, n_{2}, \\
\hat{c}_{i_{1}} & =\frac{Y_{i_{1}}}{n_{2}}-\hat{\mu}=\frac{1}{n_{2}} \sum_{i_{2}=1}^{n_{2}} Y_{i}-\hat{\mu}, \quad i_{1}=1, \ldots, n_{1} .
\end{aligned}
$$

Thus, we estimate $\mu_{i}=E Y_{\boldsymbol{i}}$ with $\hat{\mu}_{\boldsymbol{i}}=\hat{\mu}+\hat{r}_{i_{2}}+\hat{c}_{i_{1}}$.

Observe that we cannot replace the $X_{i}$-variables with the $Y_{i}$-variables in the formulas for the variance estimators (1)-(4), since then the varying mean values of the $Y_{i}$-variables will ruin the estimate of variance of $\sqrt{N} \bar{Y}$. We can, however, replace the $X_{i}$-variables with the residuals, as is done in (5).

Theorem 2 If $\mathbf{A M}^{\prime}, \mathbf{A D}(\boldsymbol{m}), \mathbf{A L}(\delta)$, and $\mathbf{A K}(\delta)$ are valid, then

$$
\hat{\gamma}_{\boldsymbol{n}}^{\left(n e^{\prime}\right)}-\gamma_{\boldsymbol{n}}^{\prime} \stackrel{P}{\longrightarrow} 0, \text { as } n_{1}, n_{2} \rightarrow \infty
$$

\section{DISCUSSION}

In Section 2 we saw that the different estimators are consistent for a broad range of choices of the rate of block size. In practice, a specific block size need to be chosen, and the choice is dependent on the numbers $\boldsymbol{n}$, and on the dependence structure. For strictly stationary data $\mathrm{X}_{n}=\left\{X_{i}: i \in \mathcal{A}_{n}\right\}$, Sherman (1996) gave a rate of convergence for his estimator of variance of 
a general statistic $s\left(\mathbf{X}_{n}\right)$ (e.g., a sample mean), and showed that this rate is minimized when the block/subshape size is proportional to $\sqrt{N}$. Similar results are obtained by Politis and Romano (1993). Further, a closely related result holds for nonstationary data, when $s\left(\mathbf{X}_{n}\right)$ is a sample mean:

Corollary $\mathbf{1}$ If $\mathbf{A M}, \mathbf{A D}(\boldsymbol{m}), \mathbf{A L}(2)$, and $\mathbf{A K}(2)$ are valid, then

$$
E\left(\hat{\gamma}_{\boldsymbol{n}}^{(n e)}-\gamma_{\boldsymbol{n}}\right)^{2} \leq C_{1}\left(K N^{-1}+k_{1}^{-2}+k_{2}^{-2}\right),
$$

for some $C_{1}>0$. Thus, if $0<\underline{\lim } n_{1} n_{2}^{-1} \leq \varlimsup \overline{\lim } n_{1} n_{2}^{-1}<\infty$ and $0<\underline{\lim } k_{i} n_{i}^{-1 / 2} \leq$ $\varlimsup k_{i} n_{i}^{-1 / 2}<\infty, i=1,2$, then

$$
\sqrt{N} E\left(\hat{\gamma}_{\boldsymbol{n}}^{(n e)}-\gamma_{\boldsymbol{n}}\right)^{2}=O(1) .
$$

Corollary 2 If $\mathbf{A M}^{\prime}, \mathbf{A D}(\boldsymbol{m}), \mathbf{A L}(2)$, and $\mathbf{A K}(2)$ are valid, then

$$
E\left(\hat{\gamma}_{\boldsymbol{n}}^{\left(n e^{\prime}\right)}-\gamma_{\boldsymbol{n}}\right)^{2} \leq C_{2}\left(K N^{-1}+k_{1}^{-2}+k_{2}^{-2}+k_{1}^{2} n_{1}^{-2}+k_{2}^{2} n_{2}^{-2}\right),
$$

for some $C_{2}>0$. Thus, if $0<\underline{\lim } n_{1} n_{2}^{-1} \leq \varlimsup \overline{\lim } n_{1} n_{2}^{-1}<\infty$ and $0<\underline{\lim } k_{i} n_{i}^{-1 / 2} \leq$ $\varlimsup k_{i} n_{i}^{-1 / 2}<\infty, i=1,2$, then

$$
\sqrt{N} E\left(\hat{\gamma}_{\boldsymbol{n}}^{\left(n e^{\prime}\right)}-\gamma_{\boldsymbol{n}}^{\prime}\right)^{2}=O(1) .
$$

It should be noted that the mean square errors (MSEs) of $\hat{\gamma}_{\boldsymbol{n}}^{(n e)}$ and $\hat{\gamma}_{\boldsymbol{n}}^{\left(n e^{\prime}\right)}$ tend to zero asymptotically, but at a rather slow rate. As noted by Politis and Romano (1993) and Sherman (1996), this is due to the relatively large contribution of bias to the MSE. Also, note that the results above only give the correct order of block size. In practice we need to choose $k_{i} \sim c_{i} n_{i}^{1 / 2}$, for some constant $c_{i}>0, i=1,2$. Clearly, the stronger the strength of dependence is, the larger values of $c_{1}$ and $c_{2}$ need to be chosen. The choice of block size in practice is indeed an important and difficult task, and only a few guidelines exist; see e.g., Politis, Romano, and Wolf (Chapter 9, 1999), who discuss this topic in the case of stationary sequences. If little is known about the dependence structure, a safe policy is to use relatively large blocks (Sherman, 1996).

All estimators of $\gamma_{\boldsymbol{n}}$ considered in this paper are biased. Consider, e.g., $\hat{\gamma}_{\boldsymbol{n}}^{(n e)}$. It is possible to write the bias of $\hat{\gamma}_{\boldsymbol{n}}^{(n e)}$ as the sum of two terms, $A_{1}$ and $A_{2}$, where $A_{1}$ is approximately equal to $-\gamma_{n} K / N$, when $K / N$ is not too "large". The second term, $A_{2}$, is negative if the observations are positively correlated, and vanishes as $K \rightarrow \infty$. $A_{2}$ is difficult to estimate; in particular for nonstationary data. In the next section, where we carry out a simulation study, the estimator $\hat{\gamma}_{\boldsymbol{n}}^{(n e c)}=\hat{\gamma}_{\boldsymbol{n}}^{(n e)}(1+K / N)$, i.e. $\hat{\gamma}_{\boldsymbol{n}}^{(n e)}$ corrected for the bias term $A_{1}$, will be considered. (From the proof of Theorem 1 it follows that 
$A_{1}=E Q_{n}^{(5)}+2 E Q_{n}^{(6)}$ and $A_{2}=2 E Q_{n}^{(3)}$, where $Q_{n}^{(3)}, Q_{n}^{(5)}$, and $Q_{n}^{(6)}$, are defined in the beginning of the proof.)

It is easily seen that the results in this paper hold also for rectangular index sets $\mathcal{A}$ expanding in all directions, i.e. for $\left\{\boldsymbol{i}: i_{1}=-n_{1}, \ldots, n_{1}\right.$ and $i_{2}=$ $\left.-n_{2}, \ldots, n_{2}\right\}$. Moreover, for all estimators of variance in Section 1, except $\hat{\gamma}_{\boldsymbol{n}}^{(c b e)}$, the assumption on the index set to be of rectangular shape can be relaxed by using "subshapes", as described in, e.g., Sherman (1996). This extention to non-rectangular $\mathcal{A}$ is not possible for $\hat{\gamma}_{n}^{(c b e)}$, since this estimator is based on the idea of "wrapping" the data around on a torus, which requires the set $\mathcal{A}$ to be of rectangular shape.

Finally, by examining the proofs of Theorem 1 and 2, we see that one can allow $m_{1}$ and $m_{2}$ to increase to infinity, as the sample size increases. (Since our proofs are not written with this in mind, i.e. of getting weak assumptions on the rate of $m_{1}$ and $m_{2}$, we do not give this as an explicit result.)

\section{A SIMULATION STUDY}

In this Monte Carlo study, nonstationary spatially $\boldsymbol{m}$-dependent data $X_{\boldsymbol{i}}$, $i \in \mathcal{A}_{n}$, are generated, where each $X_{\boldsymbol{i}}$ is a weighted average of independent and skewly distributed r.v.s such that $X_{i}$ has a small variance if both $i_{1}$ and $i_{2}$ are small, whereas the variance of $X_{\boldsymbol{i}}$ is large when $i_{1}$ and $i_{2}$ are large. To be more specific, let $m_{i}=2 l_{i}$ for some integer $l_{i} \geq 0, i=1,2$, and define weights $w(\boldsymbol{i})=v_{\boldsymbol{i}} / \sum_{j_{1}=-l_{1}}^{l_{1}} \sum_{j_{2}=-l_{2}}^{l_{2}} v_{\boldsymbol{j}}$, where $v_{\boldsymbol{i}}=\left(\left(1+\left|i_{1}\right|\right)\left(1+\left|i_{2}\right|\right)\right)^{-1}, i_{1}=-l_{1}, \ldots, l_{1}$, $i_{2}=-l_{2}, \ldots, l_{2}$. Define

$$
X_{i}=\sum_{j_{1}=i_{1}-l_{1}}^{i_{1}+l_{1}} \sum_{j_{2}=i_{2}-l_{2}}^{i_{2}+l_{2}} w\left(\left|i_{1}-j_{1}\right|,\left|i_{2}-j_{2}\right|\right)\left(Z_{j}-E Z_{j}\right)
$$

where the r.v.s $Z_{j}$ are independent and $\log$-normal with $\theta=E\left(\log Z_{j}\right)=0$, and

$$
\sigma_{j}=\sqrt{\operatorname{Var}\left(\log Z_{j}\right)}=\frac{1}{2} \sum_{i=1}^{2} \frac{j_{i}+l_{i}}{n_{i}+2 l_{i}+1}, \text { for all } \boldsymbol{j},
$$

as parameters.

In the tables below, there are columns with Monte Carlo estimates of expected values $(\widehat{E})$, standard deviations $(\sqrt{\widehat{V a r}})$, and root mean square errors $(\widehat{R M S E})$, respectively, of different estimators of $\gamma_{n}=\operatorname{Var}(\sqrt{N} \bar{X})$. In the study, $\mathcal{A}_{n}$ is a rectangular lattice of $N=250 \times 250$ points, and each Monte Carlo estimate is based on 10000 replicates. 
TABLE I. $\boldsymbol{m}=(2,2)$ and $\gamma_{\boldsymbol{n}} \approx 0.594$.

\begin{tabular}{|c||c|c|c||c|c|c||c|c|c|}
\hline \multicolumn{1}{|c||}{} & \multicolumn{3}{c||}{$K=10 \times 10$} & \multicolumn{3}{c||}{$K=20 \times 20$} & \multicolumn{3}{c|}{$K=30 \times 30$} \\
\hline & $\widehat{E}$ & $\sqrt{\widehat{V a r}}$ & $\widehat{R M S E}$ & $\widehat{E}$ & $\sqrt{\widehat{V a r}}$ & $\widehat{R M S E}$ & $\widehat{E}$ & $\sqrt{\widehat{V a r}}$ & $\widehat{R M S E}$ \\
\hline \hline$Q_{n}^{(j e)}$ & 0.494 & 0.037 & 0.106 & 0.515 & 0.063 & 0.102 & 0.508 & 0.089 & 0.124 \\
\hline$Q_{n}^{(b e)}$ & 0.495 & 0.037 & 0.105 & 0.518 & 0.064 & 0.099 & 0.515 & 0.091 & 0.121 \\
\hline$Q_{n}^{(c b e)}$ & 0.507 & 0.039 & 0.095 & 0.545 & 0.067 & 0.083 & 0.555 & 0.096 & 0.103 \\
\hline$Q_{n}^{(n e)}$ & 0.507 & 0.039 & 0.095 & 0.546 & 0.067 & 0.083 & 0.556 & 0.095 & 0.102 \\
\hline$Q_{n}^{(\text {nec })}$ & 0.508 & 0.039 & 0.095 & 0.549 & 0.068 & 0.081 & 0.564 & 0.096 & 0.101 \\
\hline$Q_{n}^{\left(n e^{\prime}\right)}$ & 0.466 & 0.036 & 0.133 & 0.465 & 0.058 & 0.141 & 0.441 & 0.077 & 0.171 \\
\hline
\end{tabular}

TABLE II. $\boldsymbol{m}=(10,10)$ and $\gamma_{\boldsymbol{n}} \approx 0.564$.

\begin{tabular}{|c||c|c|c||c|c|c||c|c|c|}
\hline \multicolumn{1}{|c|}{} & \multicolumn{3}{c||}{$K=20 \times 20$} & \multicolumn{3}{c||}{$K=30 \times 30$} & \multicolumn{3}{c|}{$K=40 \times 40$} \\
\hline & $\widehat{E}$ & $\sqrt{\widehat{V a r}}$ & $\widehat{R M S E}$ & $\widehat{E}$ & $\sqrt{\widehat{V a r}}$ & $\widehat{R M S E}$ & $\widehat{E}$ & $\sqrt{\widehat{V a r}}$ & $\widehat{R M S E}$ \\
\hline \hline$Q_{n}^{(j e)}$ & 0.405 & 0.057 & 0.169 & 0.432 & 0.084 & 0.157 & 0.437 & 0.108 & 0.167 \\
\hline$Q_{n}^{(b e)}$ & 0.409 & 0.057 & 0.166 & 0.439 & 0.086 & 0.152 & 0.449 & 0.113 & 0.161 \\
\hline$Q_{n}^{(c b e)}$ & 0.420 & 0.058 & 0.156 & 0.460 & 0.086 & 0.135 & 0.477 & 0.111 & 0.141 \\
\hline$Q_{n}^{(n e)}$ & 0.420 & 0.058 & 0.155 & 0.461 & 0.086 & 0.134 & 0.479 & 0.111 & 0.140 \\
\hline$Q_{n}^{(\text {nec })}$ & 0.423 & 0.058 & 0.153 & 0.468 & 0.087 & 0.130 & 0.492 & 0.114 & 0.135 \\
\hline$Q_{n}^{\left(n e^{\prime}\right)}$ & 0.351 & 0.049 & 0.219 & 0.359 & 0.068 & 0.216 & 0.349 & 0.084 & 0.231 \\
\hline
\end{tabular}

In Table I and II above we see that $\hat{\gamma}_{\boldsymbol{n}}^{(c b e)}$ and $\hat{\gamma}_{\boldsymbol{n}}^{(n e)}$ give close results, and that they perform better in terms of mean square error than $\hat{\gamma}_{\boldsymbol{n}}^{(j e)}$ and $\hat{\gamma}_{\boldsymbol{n}}^{(b e)}$. Although $\hat{\gamma}_{\boldsymbol{n}}^{(\text {nec })}$ is the estimator with the largest variance in the study, it compensates this with a comparatively small bias, and is the overall winner in terms of mean square error.

\section{PROOFS}

Two useful inequalities:

Inequality $\mathrm{A}$ : For any positive numbers $z_{1}, \ldots, z_{r}$ and $\lambda \geq 1$ we have, from the Jensen inequality,

$$
\left(z_{1}+\ldots+z_{r}\right)^{\lambda} \leq r^{\lambda-1}\left(z_{1}^{\lambda}+\ldots+z_{r}^{\lambda}\right)
$$

Inequality B: For any independent random variables $Z_{1}, \ldots, Z_{r}$ and $\lambda \geq 1$, with $E Z_{i}=0$ and $E\left|Z_{i}\right|^{\lambda}<\infty, i=1, \ldots, r$, we have

$$
E\left|Z_{1}+\ldots+Z_{r}\right|^{\lambda} \leq \eta r^{(\lambda / 2-1) \vee 0}\left(E\left|Z_{1}\right|^{\lambda}+\ldots+E\left|Z_{r}\right|^{\lambda}\right),
$$


where $1 \leq \eta<\infty$ is a constant, and $\eta \leq 2$ if $\lambda \leq 2$ (Petrov 1995, p. 82-83). In cases when Inequality $\mathrm{B}$ will be used repeatedly, say $k$ times and with constants $\eta_{1}, \ldots, \eta_{k}$, then we put $\eta=\max _{1 \leq i \leq k} \eta_{i}$.

Lemma 1 Assume, for all $\boldsymbol{n}$, that $\alpha_{\boldsymbol{i}}=\alpha_{\boldsymbol{i}}(\boldsymbol{n})$ has an absolute value less or equal to $1, i_{1}=a_{1}+1, \ldots, b_{1}, i_{2}=a_{2}+1, \ldots, b_{2}$, where $b_{i}-a_{i} \geq m_{i}, i=1,2$. Then, under the assumptions of Theorem 1 , for some constant $\eta \geq 1$ and $M=m_{1} m_{2}$,

(i) $\quad E\left|\sum_{i_{2}=a_{2}+1}^{b_{2}} \alpha_{i} X_{i}\right|^{2+\delta} \leq \tau_{\delta} \eta\left(8 m_{2}\left(b_{2}-a_{2}\right)\right)^{1+\delta / 2}$,

$$
E\left|\sum_{i_{1}=a_{1}+1}^{b_{1}} \sum_{i_{2}=a_{2}+1}^{b_{2}} \alpha_{i} X_{i}\right|^{2+\delta} \leq \tau_{\delta} \eta\left(64 M\left(b_{1}-a_{1}\right)\left(b_{2}-a_{2}\right)\right)^{1+\delta / 2} .
$$

Proof. We only prove (ii). Define

$$
\mathcal{M}_{j}=\left\{\left(m_{1}\left(j_{1}-1\right)+a_{1}, m_{2}\left(j_{2}-1\right)+a_{2}\right), \ldots,\left(m_{1} j_{1} \wedge b_{1}, m_{2} j_{2} \wedge b_{2}\right)\right\},
$$

$j_{1}=1, \ldots, q_{1}$ and $j_{2}=1, \ldots, q_{2}$, where $q_{i}, i=1,2$, is the largest integer such that $q_{i} \leq\left(b_{i}-a_{i}\right) / m_{i}+1$. Further, define

$$
\mathcal{D}_{\boldsymbol{g}}=\left\{\boldsymbol{j}: \mathcal{M}_{\boldsymbol{j}} \in \bigcup_{f_{1}: g_{1}+2\left(f_{1}-1\right) \leq q_{1}} \bigcup_{f_{2}: g_{2}+2\left(f_{2}-1\right) \leq q_{2}} \mathcal{M}_{g_{1}+2\left(f_{1}-1\right), g_{2}+2\left(f_{2}-1\right)}\right\} .
$$

By Inequality A with $\lambda=2+\delta$ and $r=2^{2}$, Inequality B with $\lambda=2+\delta$ and $r=\left|\mathcal{D}_{\boldsymbol{g}}\right|<q_{1} q_{2}$, and Inequality A with $\lambda=2+\delta$ and $r=\left|\mathcal{M}_{\boldsymbol{j}}\right|<M$, respectively,

$$
\begin{aligned}
E\left|\sum_{i=a_{1}+1}^{b_{1}} \sum_{i=a_{2}+1}^{b_{2}} \alpha_{\boldsymbol{i}} X_{\boldsymbol{i}}\right|^{2+\delta}=E\left|\sum_{g_{1}, g_{2}=1}^{2} \sum_{\boldsymbol{j} \in \mathcal{D}_{\boldsymbol{g}}} \sum_{\boldsymbol{i} \in \mathcal{M}_{\boldsymbol{j}}} \alpha_{\boldsymbol{i}} X_{\boldsymbol{i}}\right|^{2+\delta} \\
\leq 4^{1+\delta} \sum_{g_{1}, g_{2}=1}^{2} E\left|\sum_{\boldsymbol{j} \in \mathcal{D}_{\boldsymbol{g}}} \sum_{\boldsymbol{i} \in \mathcal{M}_{\boldsymbol{j}}} \alpha_{\boldsymbol{i}} X_{\boldsymbol{i}}\right|^{2+\delta} \\
\leq \eta 4^{1+\delta}\left(q_{1} q_{2}\right)^{\delta / 2} \sum_{g_{1}, g_{2}=1}^{2} \sum_{\boldsymbol{j} \in \mathcal{D}_{\boldsymbol{g}}} E\left|\sum_{\boldsymbol{i} \in \mathcal{M}_{\boldsymbol{j}}} \alpha_{\boldsymbol{i}} X_{\boldsymbol{i}}\right|^{2+\delta} \\
\leq \eta(4 M)^{1+\delta}\left(q_{1} q_{2}\right)^{\delta / 2} \sum_{g_{1}, g_{2}=1}^{2} \sum_{\boldsymbol{j} \in \mathcal{D}_{\boldsymbol{g}}} \sum_{\boldsymbol{i} \in \mathcal{M}_{\boldsymbol{j}}}\left|\alpha_{\boldsymbol{i}}\right|^{2+\delta} E\left|X_{\boldsymbol{i}}\right|^{2+\delta} \\
\leq \tau_{\delta} \eta(4 M)^{2+\delta}\left(q_{1} q_{2}\right)^{1+\delta / 2} \\
\leq \tau_{\delta} \eta(4 M)^{2+\delta}\left(\frac{b_{1}-a_{1}}{m_{1}}+1\right)^{1+\delta / 2}\left(\frac{b_{2}-a_{2}}{m_{2}}+1\right)^{1+\delta / 2}
\end{aligned}
$$


from which the desired result follows.

Proof of Theorem 1. Throughout the proof we will, without loss of generality, assume that $\mu=0$ and $n_{i}>k_{i}>m_{i} \geq 1, i=1,2$. Define $X_{i}=0$ whenever $\boldsymbol{i} \notin \mathcal{A}_{\boldsymbol{n}}$ and $\delta^{\prime}=1+\delta / 2$. Then,

$$
\begin{aligned}
& \hat{\gamma}_{\boldsymbol{n}}=\hat{\gamma}_{\boldsymbol{n}}^{(n e)}=\frac{1}{K N} \sum_{\boldsymbol{i} \in \mathcal{A}_{\boldsymbol{n}}^{\prime}}\left(\sum_{\boldsymbol{j} \in B_{\boldsymbol{i}}}\left(X_{\boldsymbol{j}}-\frac{X . .}{N}\right) I_{j}\right)^{2} \\
& =\frac{1}{K N} \sum_{i \in \mathcal{A}_{n}^{\prime}}\left(\sum_{j \in B_{i}} X_{j}\right)^{2}+\frac{X^{2} \cdot}{K N^{3}} \sum_{i \in \mathcal{A}_{n}^{\prime}}\left(\sum_{j \in B_{i}} I_{j}\right)^{2} \\
& -\frac{2 X . .}{K N^{2}} \sum_{i \in \mathcal{A}_{n}^{\prime}}\left(\sum_{j \in B_{i}} X_{j}\right)\left(\sum_{j \in B_{i}} I_{j}\right) \\
& =\frac{1}{N} \sum_{h_{1}=1-k_{1}}^{k_{1}-1} \sum_{h_{2}=1-k_{2}}^{k_{2}-1} \sum_{i_{1}=1-0 \wedge h_{1}}^{n_{1}-0 \vee h_{1}} \sum_{i_{2}=1-0 \wedge h_{2}}^{n_{2}-0 \vee h_{2}} X_{\boldsymbol{i}} X_{\boldsymbol{i}+\boldsymbol{h}} w_{h_{1}} w_{h_{2}} \\
& +\frac{X^{2}}{N^{3}} \sum_{h_{1}=1-k_{1}}^{k_{1}-1} \sum_{h_{2}=1-k_{2}}^{k_{2}-1} \sum_{i_{1}=1-0 \wedge h_{1}}^{n_{1}-0 \vee h_{1}} \sum_{i_{2}=1-0 \wedge h_{2}}^{n_{2}-0 \vee h_{2}} w_{h_{1}} w_{h_{2}} \\
& -\frac{2 X . .}{N^{2}} \sum_{h_{1}=1-k_{1}}^{k_{1}-1} \sum_{h_{2}=1-k_{2}}^{k_{2}-1} \sum_{i_{1}=1-0 \wedge h_{1}}^{n_{1}-0 \vee h_{1}} \sum_{i_{2}=1-0 \wedge h_{2}}^{n_{2}-0 \vee h_{2}} X_{\boldsymbol{i}} w_{h_{1}} w_{h_{2}} \text {, }
\end{aligned}
$$

where $w_{h_{i}}=\left(k_{i}-\left|h_{i}\right|\right) / k_{i}, i=1,2$. Let $\boldsymbol{h}_{0}=\left(0, h_{2}\right)$. By AM (with $\left.\mu=0\right)$ and $\operatorname{AD}(m)$

$$
\begin{aligned}
\gamma_{\boldsymbol{n}}= & \frac{1}{N} \sum_{\boldsymbol{i} \in \mathcal{A}_{\boldsymbol{n}}} E X_{\boldsymbol{i}}^{2}+\frac{2}{N} \sum_{h_{2}=1}^{m_{2}} \sum_{i_{1}=1}^{n_{1}} \sum_{i_{2}=1}^{n_{2}-h_{2}} E X_{\boldsymbol{i}} X_{\boldsymbol{i}+\boldsymbol{h}_{0}} \\
& +\frac{2}{N} \sum_{h_{1}=1}^{m_{1}} \sum_{h_{2}=-m_{2}}^{m_{2}} \sum_{i_{1}=1}^{n_{1}-h_{1}} \sum_{i_{2}=1-0 \wedge h_{2}}^{n_{2}-0 \vee h_{2}} E X_{\boldsymbol{i}} X_{\boldsymbol{i}+\boldsymbol{h}} .
\end{aligned}
$$

Let $U_{\boldsymbol{i}, \boldsymbol{h}}=X_{\boldsymbol{i}} X_{\boldsymbol{i}+\boldsymbol{h}}$ and $V_{\boldsymbol{i}, \boldsymbol{h}}=U_{\boldsymbol{i}, \boldsymbol{h}}-E U_{\boldsymbol{i}, \boldsymbol{h}}$. By combining (6) and (7), we get

$$
\hat{\gamma}_{\boldsymbol{n}}-\gamma_{\boldsymbol{n}}=Q_{\boldsymbol{n}}^{(1)}+2 Q_{\boldsymbol{n}}^{(2)}+2 Q_{\boldsymbol{n}}^{(3)}+2 Q_{\boldsymbol{n}}^{(4)}+Q_{\boldsymbol{n}}^{(5)}+2 Q_{n}^{(6)}
$$

where

$$
Q_{n}^{(1)}=\frac{1}{N} \sum_{i \in \mathcal{A}_{n}}\left(X_{i}^{2}-E X_{i}^{2}\right)
$$




$$
\begin{aligned}
& Q_{n}^{(2)}=\frac{1}{N} \sum_{h_{2}=1}^{m_{2}} \sum_{i_{1}=1}^{n_{1}} \sum_{i_{2}=1}^{n_{2}-h_{2}} V_{\boldsymbol{i}, \boldsymbol{h}_{0}}+\frac{1}{N} \sum_{h_{1}=1}^{m_{1}} \sum_{h_{2}=-m_{2}}^{m_{2}} \sum_{i_{1}=1}^{n_{1}-h_{1}} \sum_{i_{2}=1-0 \wedge h_{2}}^{n_{2}-0 \vee h_{2}} V_{\boldsymbol{i}, \boldsymbol{h}}, \\
& Q_{n}^{(3)}=-\frac{1}{N} \sum_{h_{2}=1}^{m_{2}} \sum_{i_{1}=1}^{n_{1}} \sum_{i_{2}=1}^{n_{2}-h_{2}} U_{i, h_{0}} \frac{h_{2}}{k_{2}} \\
& +\frac{1}{N} \sum_{h_{1}=1}^{m_{1}} \sum_{h_{2}=-m_{2}}^{m_{2}} \sum_{i_{1}=1}^{n_{1}-h_{1}} \sum_{i_{2}=1-0 \wedge h_{2}}^{n_{2}-0 \vee h_{2}} U_{i, h} \frac{h_{1}\left|h_{2}\right|-h_{1} k_{2}-\left|h_{2}\right| k_{1}}{K}, \\
& Q_{n}^{(4)}=\frac{1}{N} \sum_{h_{2}=m_{2}+1}^{k_{2}-1} \sum_{i_{1}=1}^{n_{1}} \sum_{i_{2}=1}^{n_{2}-h_{2}} U_{\boldsymbol{i}, \boldsymbol{h}_{0}} w_{h_{2}} \\
& +\frac{1}{N} \sum_{h_{1}=m_{1}+1}^{k_{1}-1} \sum_{h_{2}=1-k_{2}}^{k_{2}-1} \sum_{i_{1}=1}^{n_{1}-h_{1}} \sum_{i_{2}=1-0 \wedge h_{2}}^{n_{2}-0 \vee h_{2}} U_{i, h} w_{h_{1}} w_{h_{2}} \\
& +\frac{1}{N} \sum_{h_{1}=1}^{m_{1}}\left(\sum_{h_{2}=1-k_{2}}^{-m_{2}-1}+\sum_{h_{2}=m_{2}+1}^{k_{2}-1}\right) \sum_{i_{1}=1}^{n_{1}-h_{1}} \sum_{i_{2}=1-0 \wedge h_{2}}^{n_{2}-0 \vee h_{2}} U_{i, h} w_{h_{1}} w_{h_{2}}, \\
& Q_{n}^{(5)}=\frac{X_{2}^{2}}{N^{3}} \sum_{h_{1}=1-k_{1}}^{k_{1}-1} \sum_{h_{2}=1-k_{2}}^{k_{2}-1} \sum_{i_{1}=1-0 \wedge h_{1}}^{n_{1}-0 \vee h_{1}} \sum_{i_{2}=1-0 \wedge h_{2}}^{n_{2}-0 \vee h_{2}} w_{h_{1}} w_{h_{2}} \text {, } \\
& Q_{n}^{(6)}=-\frac{X . .}{N^{2}} \sum_{h_{1}=1-k_{1}}^{k_{1}-1} \sum_{h_{2}=1-k_{2}}^{k_{2}-1} \sum_{i_{1}=1-0 \wedge h_{1}}^{n_{1}-0 \vee h_{1}} \sum_{i_{2}=1-0 \wedge h_{2}}^{n_{2}-0 \vee h_{2}} X_{i} w_{h_{1}} w_{h_{2}} \text {. }
\end{aligned}
$$

Write $Q_{n}^{(2)}=Q_{n}^{(2 a)}+Q_{n}^{(2 b)}+Q_{n}^{(2 c)}$, where

$$
\begin{aligned}
Q_{n}^{(2 a)} & =\sum_{h_{2}=1}^{m_{2}} \sum_{i_{1}=1}^{n_{1}} \sum_{i_{2}=1}^{n_{2}-h_{2}} \frac{V_{\boldsymbol{i}, \boldsymbol{h}_{0}}}{N}, \\
Q_{n}^{(2 b)} & =\sum_{h_{1}=1}^{m_{1}} \sum_{h_{2}=1}^{m_{2}} \sum_{i_{1}=1}^{n_{1}-h_{1}} \sum_{i_{2}=1}^{n_{2}-h_{2}} \frac{V_{\boldsymbol{i}, \boldsymbol{h}}}{N}, \\
Q_{\boldsymbol{n}}^{(2 c)} & =\sum_{h_{1}=1}^{m_{1}} \sum_{h_{2}=-m_{2}}^{0} \sum_{i_{1}=1}^{n_{1}-h_{1}} \sum_{i_{2}=1-h_{2}}^{n_{2}} \frac{V_{\boldsymbol{i}, \boldsymbol{h}}}{N} .
\end{aligned}
$$

Consider $Q_{\boldsymbol{n}}^{(2 b)}$. By Inequality A, with $\lambda=\delta^{\prime}$ and $r=M=m_{1} m_{2}$,

$$
E\left|Q_{\boldsymbol{n}}^{(2 b)}\right|^{\delta^{\prime}} \leq M^{\delta / 2} \sum_{h_{1}=1}^{m_{1}} \sum_{h_{2}=1}^{m_{2}} E\left|\sum_{i_{1}=1}^{n_{1}-h_{1}} \sum_{i_{2}=1}^{n_{2}-h_{2}} \frac{V_{\boldsymbol{i}, \boldsymbol{h}}}{N}\right|^{\delta^{\prime}} .
$$

Define

$$
\mathcal{M}_{j}^{\prime}=\left\{\left(m_{1}\left(j_{1}-1\right)+1, m_{2}\left(j_{2}-1\right)+1\right), \ldots,\left(m_{1} j_{1} \wedge n_{1}, m_{2} j_{2} \wedge n_{2}\right)\right\},
$$


$j_{1}=1, \ldots, q_{1}^{\prime}$ and $j_{2}=1, \ldots, q_{2}^{\prime}$, where $q_{i}^{\prime}, i=1,2$, is the largest integer such that $q_{i}^{\prime} \leq n_{i} / m_{i}+1$. Further, define

$$
\mathcal{D}_{\boldsymbol{g}}^{\prime}=\left\{\boldsymbol{j}: \mathcal{M}_{\boldsymbol{j}}^{\prime} \in \bigcup_{f_{1}: g_{1}+3\left(f_{1}-1\right) \leq q_{1}^{\prime} f_{2}: g_{2}+3\left(f_{2}-1\right) \leq q_{2}^{\prime}} \bigcup_{g_{1}+3\left(f_{1}-1\right), g_{2}+3\left(f_{2}-1\right)}^{\prime}\right\} .
$$

By Inequality A with $\lambda=\delta^{\prime}$ and $r=3^{2}$, Inequality B with $\lambda=\delta^{\prime}$ and $r=\left|\mathcal{D}_{\boldsymbol{g}}^{\prime}\right|$, Inequality $\mathrm{A}$ with $\lambda=\delta^{\prime}$ and $r=M=m_{1} m_{2}$, and Inequality $\mathrm{A}$ with $\lambda=\delta^{\prime}$ and $r=2$, respectively,

$$
\begin{aligned}
& E\left|\sum_{i_{1}=1}^{n_{1}-h_{1}} \sum_{i_{2}=1}^{n_{2}-h_{2}} \frac{V_{\boldsymbol{i}, \boldsymbol{h}}}{N}\right|^{\delta^{\prime}}=E\left|\sum_{g_{1}, g_{2}=1}^{3} \sum_{\boldsymbol{j} \in \mathcal{D}_{\boldsymbol{g}}^{\prime}} \sum_{\boldsymbol{i} \in \mathcal{M}_{\boldsymbol{j}}^{\prime}} \frac{V_{\boldsymbol{i}, \boldsymbol{h}}}{N}\right|^{\delta^{\prime}} \\
& \leq 3^{\delta} \sum_{g_{1}, g_{2}=1}^{3} E\left|\sum_{\boldsymbol{j} \in \mathcal{D}_{\boldsymbol{g}}^{\prime}} \sum_{\boldsymbol{i} \in \mathcal{M}_{\boldsymbol{j}}^{\prime}} \frac{V_{\boldsymbol{i}, \boldsymbol{h}}}{N}\right|^{\delta^{\prime}} \\
& \leq 3^{\delta} 2 \sum_{g_{1}, g_{2}=1}^{3} \sum_{\boldsymbol{j} \in \mathcal{D}_{\boldsymbol{g}}^{\prime}} E\left|\sum_{\boldsymbol{i} \in \mathcal{M}_{\boldsymbol{j}}^{\prime}} \frac{V_{\boldsymbol{i}, \boldsymbol{h}}}{N}\right|^{\delta^{\prime}} \\
& \leq 3^{\delta} 2 \sum_{g_{1}, g_{2}=1}^{3} \sum_{\boldsymbol{j} \in \mathcal{D}_{\boldsymbol{g}}^{\prime}} E\left(\sum_{\boldsymbol{i} \in \mathcal{M}_{\boldsymbol{j}}^{\prime}} \mid \frac{V_{\boldsymbol{i}, \boldsymbol{h}}}{N}\right)^{\delta^{\prime}} \\
& \leq 3^{\delta} 2 M^{\delta / 2} \sum_{g_{1}, g_{2}=1}^{3} \sum_{\boldsymbol{j} \in \mathcal{D}_{\boldsymbol{g}}^{\prime}} \sum_{\boldsymbol{i} \in \mathcal{M}_{\boldsymbol{j}}^{\prime}} E\left|\frac{V_{\boldsymbol{i}, \boldsymbol{h}}}{N}\right|^{\delta^{\prime}} \\
& \leq 3^{\delta} 2^{\delta^{\prime}} M^{\delta / 2} \sum_{g_{1}, g_{2}=1}^{3} \sum_{\boldsymbol{j} \in \mathcal{D}_{\boldsymbol{g}}^{\prime}} \sum_{\boldsymbol{i} \in \mathcal{M}_{\boldsymbol{j}}^{\prime}} \frac{E\left|U_{\boldsymbol{i}, \boldsymbol{h}}\right|^{\delta^{\prime}}+\left|E U_{\boldsymbol{i}, \boldsymbol{h}}\right|^{\delta^{\prime}}}{N^{\delta^{\prime}}} \\
& \leq 3^{\delta} 2^{2 \delta^{\prime} / 2} M^{\delta / 2}\left(E\left|X_{\boldsymbol{i}}\right|^{\delta^{\prime}} E\left|X_{\boldsymbol{i}+\boldsymbol{h}}\right|^{2 \delta^{\prime}}\right)^{1 / 2} N^{-\delta / 2} \\
& \leq 4 \tau_{\delta}(18 M / N)^{\delta / 2} .
\end{aligned}
$$

By combining inequalities (9) and (10), $E\left|Q_{n}^{(2 b)}\right|^{\delta^{\prime}} \leq 4 \tau_{\delta} M\left(18 M^{2} / N\right)^{\delta / 2} \rightarrow 0$, as $n_{1}, n_{2} \rightarrow \infty$. Likewise, $E\left|Q_{n}^{(2 a)}\right|^{\delta^{\prime}} \rightarrow 0$ and $E\left|Q_{n}^{(2 c)}\right|^{\delta^{\prime}} \rightarrow 0$, and thus, by the Chebyshev inequality,

$$
Q_{n}^{(2)} \stackrel{P}{\longrightarrow} 0, \text { as } n_{1}, n_{2} \rightarrow \infty .
$$

By using the same approach as above,

$$
Q_{n}^{(1)} \stackrel{P}{\longrightarrow} 0, \text { as } n_{1}, n_{2} \rightarrow \infty \text {. }
$$


Recall that $k_{i}=k_{i}\left(n_{i}\right) \rightarrow \infty$ as $n_{i} \rightarrow \infty, i=1,2$. By the Cauchy-Schwarz and the Lyapunov inequalities, respectively,

$$
\begin{aligned}
E\left|Q_{\boldsymbol{n}}^{(3)}\right| \leq & \sum_{h_{2}=1}^{m_{2}} \sum_{i_{1}=1}^{n_{1}} \sum_{i_{2}=1}^{n_{2}-h_{2}} E\left|U_{\boldsymbol{i}, \boldsymbol{h}_{0}}\right| \frac{h_{2}}{k_{2} N} \\
& +\sum_{h_{1}=1}^{m_{1}} \sum_{h_{2}=-m_{2}}^{m_{2}} \sum_{i_{1}=1}^{n_{1}-h_{1}} \sum_{i_{2}=1-0 \wedge h_{2}}^{n_{2}-0 \vee h_{2}} E\left|U_{\boldsymbol{i}, \boldsymbol{h}}\right| \frac{h_{1} k_{2}+\left|h_{2}\right| k_{1}}{K N} \\
\leq & \sum_{h_{2}=1}^{m_{2}} \sum_{i_{1}=1}^{n_{1}} \sum_{i_{2}=1}^{n_{2}-h_{2}}\left(E X_{\boldsymbol{i}}^{2} E X_{\boldsymbol{i}+\boldsymbol{h}_{0}}^{2}\right)^{1 / 2} \frac{h_{2}}{k_{2} N} \\
& +\sum_{h_{1}=1}^{m_{1}} \sum_{h_{2}=-m_{2}}^{m_{2}} \sum_{i_{1}=1}^{n_{1}-h_{1}} \sum_{i_{2}=1-0 \wedge h_{2}}^{n_{2}-0 \vee h_{2}}\left(E X_{\boldsymbol{i}}^{2} E X_{\boldsymbol{i}+\boldsymbol{h}}^{2}\right)^{1 / 2} \frac{h_{1} k_{2}+\left|h_{2}\right| k_{1}}{K N} \\
\leq & m_{2}^{2} \tau_{\delta}^{2 /\left(2 \delta^{\prime}\right)} / k_{2}+3 M \tau_{\delta}^{2 /\left(2 \delta^{\prime}\right)}\left(m_{1} k_{2}+m_{2} k_{1}\right) / K \rightarrow 0, \text { as } n_{1}, n_{2} \rightarrow \infty .
\end{aligned}
$$

Thus, by the Chebyshev inequality,

$$
Q_{n}^{(3)} \stackrel{P}{\longrightarrow} 0 \text {, as } n_{1}, n_{2} \rightarrow \infty .
$$

Consider $Q_{n}^{(4)}$. Write $Q_{n}^{(4)}=Q_{n}^{(4 a)}+Q_{n}^{(4 b)}+Q_{n}^{(4 c)}+Q_{n}^{(4 d)}+Q_{n}^{(4 e)}$, where

$$
\begin{aligned}
Q_{n}^{(4 a)} & =\frac{1}{N} \sum_{h_{2}=m_{2}+1}^{k_{2}-1} \sum_{i_{1}=1}^{n_{1}} \sum_{i_{2}=1}^{n_{2}-h_{2}} U_{\boldsymbol{i}, \boldsymbol{h}_{0}} w_{h_{2}}, \\
Q_{\boldsymbol{n}}^{(4 b)} & =\frac{1}{N} \sum_{h_{1}=m_{1}+1}^{k_{1}-1} \sum_{h_{2}=1}^{k_{2}-1} \sum_{i_{1}=1}^{n_{1}-h_{1}} \sum_{i_{2}=1}^{n_{2}-h_{2}} U_{\boldsymbol{i}, \boldsymbol{h}} w_{h_{1}} w_{h_{2}}, \\
Q_{\boldsymbol{n}}^{(4 c)} & =\frac{1}{N} \sum_{h_{1}=m_{1}+1}^{k_{1}-1} \sum_{h_{2}=1-k_{2}}^{0} \sum_{i_{1}=1}^{n_{1}-h_{1}} \sum_{i_{2}=1-h_{2}}^{n_{2}} U_{\boldsymbol{i}, \boldsymbol{h}} w_{h_{1}} w_{h_{2}}, \\
Q_{\boldsymbol{n}}^{(4 d)} & =\frac{1}{N} \sum_{h_{1}=1}^{m_{1}} \sum_{h_{2}=m_{2}+1}^{k_{2}-1} \sum_{i_{1}=1}^{n_{1}-h_{1}} \sum_{i_{2}=1}^{n_{2}-h_{2}} U_{\boldsymbol{i}, \boldsymbol{h}} w_{h_{1}} w_{h_{2}}, \\
Q_{\boldsymbol{n}}^{(4 e)} & =\frac{1}{N} \sum_{h_{1}=1}^{m_{1}} \sum_{h_{2}=1-k_{2}}^{-m_{2}-1} \sum_{n_{1}=1}^{n_{1}-h_{1}} \sum_{i_{2}=1-h_{2}}^{n_{2}} U_{\boldsymbol{i}, \boldsymbol{h}} w_{h_{1}} w_{h_{2}} .
\end{aligned}
$$

Next it will shown that $E\left|Q_{\boldsymbol{n}}^{(4 b)}\right|^{\delta^{\prime}} \rightarrow 0$, as $n_{1}, n_{2} \rightarrow \infty$. Define

$$
\mathcal{K}_{j}=\left\{\left(k_{1}\left(j_{1}-1\right)+1, k_{2}\left(j_{2}-1\right)+1\right), \ldots,\left(k_{1} j_{1} \wedge n_{1}, k_{2} j_{2} \wedge n_{2}\right)\right\},
$$

$j_{1}=1, \ldots, p_{1}$ and $j_{2}=1, \ldots, p_{2}$, where $p_{i}, i=1,2$, is the largest integer such that $p_{i} \leq n_{i} / k_{i}+1$. Further, define

$$
\mathcal{E}_{\boldsymbol{g}}=\left\{\boldsymbol{j}: \mathcal{K}_{\boldsymbol{j}} \in \bigcup_{f_{1}: g_{1}+3\left(f_{1}-1\right) \leq p_{1} f_{2}: g_{2}+3\left(f_{2}-1\right) \leq p_{2}} \mathcal{K}_{g_{1}+3\left(f_{1}-1\right), g_{2}+3\left(f_{2}-1\right)}\right\},
$$


and

$\mathcal{I}_{j_{1}}^{(1)}=\left\{\left(i_{1}, l_{1}\right): i_{1}=k_{1}\left(j_{1}-1\right)+1, \ldots, k_{1} j_{1} \wedge n_{1}, l_{1}=i_{1}+m_{1}+1, \ldots,\left(i_{1}+k_{1}-1\right) \wedge n_{1}\right\}$, $\mathcal{I}_{j_{2}}^{(2)}=\left\{\left(i_{2}, l_{2}\right): i_{2}=k_{2}\left(j_{2}-2\right)+1, \ldots, k_{2} j_{2} \wedge n_{2}, l_{2}=i_{2}+1, \ldots,\left(i_{2}+k_{2}-1\right) \wedge n_{2}\right\}$.

Then, if we define

$$
W_{j}=\sum_{\left(i_{1}, l_{1}\right) \in \mathcal{I}_{j_{1}}^{(1)}} \sum_{\left(i_{2}, l_{2}\right) \in \mathcal{I}_{j_{2}}^{(2)}} \frac{\left(k_{1}-\left(l_{1}-i_{1}\right)\right)\left(k_{2}-\left(l_{2}-i_{2}\right)\right)}{K N} X_{\boldsymbol{i}} X_{\boldsymbol{l}},
$$

we can write

$$
Q_{n}^{(4 b)}=\sum_{g_{1}, g_{2}=1}^{3} \sum_{\boldsymbol{j} \in \mathcal{E} \boldsymbol{g}} W_{\boldsymbol{j}}
$$

Since $l_{1}>i_{1}+m_{1}$ for all $\left(i_{1}, l_{1}\right) \in \mathcal{I}_{j_{1}}^{(1)}$ we have $E W_{j}=0$ for all $\boldsymbol{j}$. By Inequality A with $\lambda=\delta^{\prime}$ and $r=3^{2}$, and Inequality $\mathrm{B}$ with $\lambda=\delta^{\prime}$ and $r=\left|\mathcal{E}_{\boldsymbol{g}}\right|$,

$$
E\left|Q_{\boldsymbol{n}}^{(4 b)}\right|^{\delta^{\prime}} \leq 3^{\delta} 2 \sum_{g_{1}, g_{2}=1}^{3} \sum_{\boldsymbol{j} \in \mathcal{E}_{\boldsymbol{g}}} E\left|W_{\boldsymbol{j}}\right|^{\delta^{\prime}}
$$

All the $E\left|W_{j}\right|^{\delta^{\prime}}$ can be handled similarly, and therefore only the case $\boldsymbol{j}=\mathbf{1}$ (i.e. $j_{1}=j_{2}=1$ ) will be considered in detail below.

Split the set $\mathcal{I}_{1}^{(1)}$ into two parts,

$$
\mathcal{I}_{12}^{(1)}=\left\{\left(i_{1}, l_{1}\right): l_{1}-m_{1}-1 \geq k_{1},\left(i_{1}, l_{1}\right) \in \mathcal{I}_{1}^{(1)}\right\} \text { and } \mathcal{I}_{11}^{(1)}=\mathcal{I}_{1}^{(1)} \backslash \mathcal{I}_{12}^{(1)} .
$$

Next, $\mathcal{I}_{11}^{(1)}$ will be partioned into disjoint sets. Define

$$
\mathcal{I}_{1111}^{(1)}=\left\{\left(i_{1}, l_{1}\right): 0<i_{1} \leq\left[\frac{k_{1}}{2}\right],\left[\frac{k_{1}}{2}\right] \leq l_{1}-m_{1}-1<k_{1},\left(i_{1}, l_{1}\right) \in \mathcal{I}_{11}^{(1)}\right\}
$$

where $[x]$ denotes the greatest integer less than or equal to $x$, and

$$
\begin{aligned}
\mathcal{I}_{11 b c}^{(1)}= & \left\{\left(i_{1}, l_{1}\right):\left[\frac{(2 c-2) k_{1}}{2^{b}}\right]<i_{1} \leq\left[\frac{(2 c-1) k_{1}}{2^{b}}\right],\right. \\
& {\left.\left[\frac{(2 c-1) k_{1}}{2^{b}}\right] \leq l_{1}-m_{1}-1<\left[\frac{2 c k_{1}}{2^{b}}\right]\right\}, }
\end{aligned}
$$

for all $(b, c) \in \mathcal{C}_{11}=\left\{(b, c)\right.$ : at least one side of the "rectangle" $\mathcal{I}_{11 b c}^{(1)}$ is larger than or equal to $m_{1}, b=2,3, \ldots$, and $\left.c=1, \ldots, 2^{b-1}\right\}$. For instance, the set $\mathcal{I}_{1121}^{(1)}$ consists of $\left[k_{1} / 4\right] \times\left(\left[k_{1} / 2\right]-\left[k_{1} / 4\right]\right)$ pairs of indices $\left(i_{1}, l_{1}\right)$, and has side lengths 
$\left[k_{1} / 4\right]$ and $\left[k_{1} / 2\right]-\left[k_{1} / 4\right]$. Observe that $\mathcal{I}_{1111}^{(1)}$ does not have a rectangular shape (see Fig. 1). Define $b_{1}=\max \left\{b:(b, c) \in \mathcal{C}_{11}\right\}$. Note that all sides of all the rectangular shaped sets $\mathcal{I}_{11 b c}^{(1)}$ are less than or equal to $k_{1} / 2^{b}+1$, and that $2 m_{1} \leq k_{1} / 2^{b_{1}-1}+1$. Hence, we get the following upper bound for $b_{1}$,

$$
b_{1} \leq \log _{2} \frac{k_{1}}{2 m_{1}-1}+1 \leq \log _{2} k_{1}+1=\log _{2}\left(2 k_{1}\right) \text {. }
$$

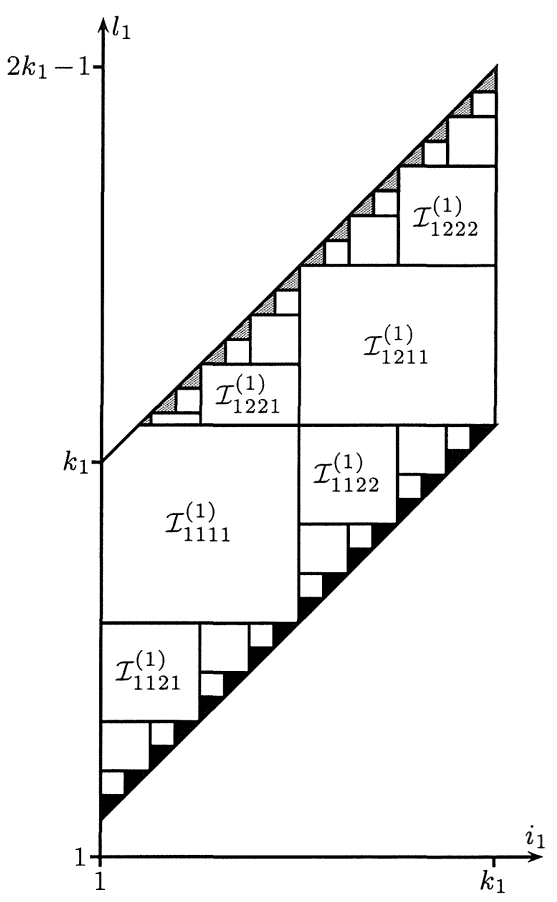

FIG. 1. Partition of $\mathcal{I}_{1}^{(1)}$

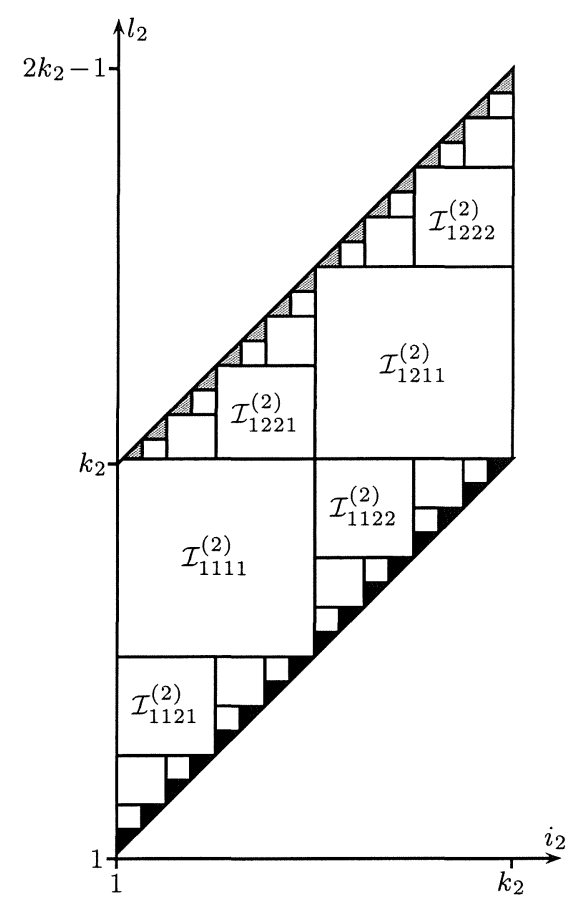

FIG. 2. Partition of $\mathcal{I}_{1}^{(2)}$

For each $b$, the number of $\mathcal{I}_{11 b c}^{(1)}$ is not more than $2^{b-1}$. For the sake of simplicity, we assume that this number is exactly $2^{b-1}$. After at most $b_{1}$ steps, we obtain our partition of $\mathcal{I}_{11}^{(1)}$,

$$
\mathcal{I}_{11}^{(1)}=\left(\bigcup_{b=1}^{b_{1}} \bigcup_{c=1}^{2^{b-1}} \mathcal{I}_{11 b c}^{(1)}\right) \bigcup\left(\bigcup_{c=1}^{2^{b_{1}}} \mathcal{I}_{11\left(b_{1}+1\right) c}^{(1)}\right)
$$

where $\mathcal{I}_{11\left(b_{1}+1\right) c}^{(1)}, c=1, \ldots, 2^{b_{1}}$, are disjoint "triangles" (the black triangles in Fig. 1), with orthogonal sides less than or equal to $2 m_{1}+1$. Similarly, we get a partition of $\mathcal{I}_{12}^{(1)}$,

$$
\mathcal{I}_{12}^{(1)}=\left(\bigcup_{b=1}^{b_{2}} \bigcup_{c=1}^{2^{b-1}} \mathcal{I}_{12 b c}^{(1)}\right) \bigcup\left(\bigcup_{c=1}^{2^{b_{2}}} \mathcal{I}_{12\left(b_{2}+1\right) c}^{(1)}\right)
$$


where $\mathcal{I}_{12 b c}^{(1)}, c=1, \ldots, 2^{b-1}, b=1, \ldots, b_{2}$, are disjoint "rectangles" with sides less than or equal to $k_{1} / 2^{b}+1$, and $\mathcal{I}_{12\left(b_{2}+1\right) c}^{(1)}, c=1, \ldots, 2^{b_{2}}$, are disjoint "triangles" (the grey triangles in Fig. 1), with orthogonal sides less than or equal to $2 m_{1}+1$.

By using the same technique, we obtain a partition of $\mathcal{I}_{1}^{(2)}$ :

$$
\left(\bigcup_{u=1}^{u_{1}} \bigcup_{v=1}^{2^{u-1}} \mathcal{I}_{11 u v}^{(2)}\right) \bigcup\left(\bigcup_{v=1}^{2^{u_{1}}} \mathcal{I}_{11\left(u_{1}+1\right) v}^{(2)}\right) \bigcup\left(\bigcup_{u=1}^{u_{2}} \bigcup_{v=1}^{2^{u-1}} \mathcal{I}_{12 u v}^{(2)}\right) \bigcup\left(\bigcup_{v=1}^{2^{u_{2}}} \mathcal{I}_{12\left(u_{2}+1\right) v}^{(2)}\right)
$$

where $\mathcal{I}_{11 u v}^{(2)}, v=1, \ldots, 2^{u-1}, u=2, \ldots, u_{1}$, and $\mathcal{I}_{12 u v}^{(2)}, v=1, \ldots, 2^{u-1}, u=1, \ldots, u_{2}$, are disjoint "rectangles" with sides less than or equal to $k_{2} / 2^{u}+1$, and $\mathcal{I}_{11\left(u_{1}+1\right) v}^{(2)}$, $v=1, \ldots, 2^{u_{1}}$, and $\mathcal{I}_{12\left(u_{2}+1\right) v}^{(2)}, v=1, \ldots, 2^{u_{2}}$, are disjoint "triangles" (the black and grey triangles, respectively, in Fig. 2), with orthogonal sides less than or equal to $2 m_{2}+1$. Just like $\mathcal{I}_{1111}^{(1)}$, the set $\mathcal{I}_{1111}^{(2)}$ is not "rectangular" (see Fig. 2).

Define

$$
W_{1 a b c}^{1 t u v}=\sum_{\left(i_{1}, l_{1}\right) \in \mathcal{I}_{1 a b c}^{(1)}} \sum_{\left(i_{2}, l_{2}\right) \in \mathcal{I}_{1 t u v}^{(2)}} \frac{\left(k_{1}-\left(l_{1}-i_{1}\right)\right)\left(k_{2}-\left(l_{2}-i_{2}\right)\right)}{K N} X_{\boldsymbol{i}} X_{\boldsymbol{l}} .
$$

To keep the notation manageable, we will write $W_{a b c}^{t u v}$ instead of $W_{1 a b c}^{1 t u v}$. Then

$$
\begin{aligned}
W_{\mathbf{1}}= & \sum_{a, t=1}^{2}\left(\sum_{b=1}^{b_{a}} \sum_{c=1}^{2^{b-1}} \sum_{u=1}^{u_{t}} \sum_{v=1}^{2^{u-1}} W_{a b c}^{t u v}\right. \\
& +\sum_{b=1}^{b_{a}} \sum_{c=1}^{2^{c-1}} \sum_{v=1}^{2^{u_{t}-1}} W_{a b c}^{t\left(u_{t}+1\right)(2 v-1)}+\sum_{b=1}^{b_{a}} \sum_{c=1}^{2^{b-1}} \sum_{v=1}^{2^{u_{t}-1}} W_{a b c}^{t\left(u_{t}+1\right)(2 v)} \\
& +\sum_{c=1}^{2^{b_{a}-1}} \sum_{u=1}^{u_{t}} \sum_{v=1}^{2^{u-1}} W_{a\left(b_{a}+1\right)(2 c-1)}^{t u v}+\sum_{c=1}^{2^{b_{a}-1}} \sum_{u=1}^{u_{t}} \sum_{v=1}^{2^{u-1}} W_{a\left(b_{a}+1\right)(2 c)}^{t u v} \\
& +\sum_{c=1}^{2^{b_{a}-1}} \sum_{v=1}^{2^{u_{t}-1}} W_{a\left(b_{a}+1\right)(2 c-1)}^{t\left(u_{t}+1\right)(2 v-1)}+\sum_{c=1}^{2^{b_{a}-1}} \sum_{v=1}^{2^{u_{t}-1}} W_{a\left(b_{a}+1\right)(2 c)}^{t\left(u_{t}+1\right)(2 v-1)} \\
& \left.+\sum_{c=1}^{2^{b_{a}-1}} \sum_{v=1}^{2^{u_{t}-1}} W_{a\left(b_{a}+1\right)(2 c-1)}^{t\left(u_{t}+1\right)(2 v)}+\sum_{c=1}^{2^{b_{a}-1}} \sum_{v=1}^{2^{u_{t}-1}} W_{a\left(b_{a}+1\right)(2 c)}^{t\left(u_{t}+1\right)(2 v)}\right) .
\end{aligned}
$$

By applying Inequality A, with $\lambda=\delta^{\prime}$ and various values of $r$, we get

$$
E\left|W_{\mathbf{1}}\right|^{\delta^{\prime}} \leq 6^{\delta} \sum_{a, t=1}^{2}\left(\left(b_{a} u_{t}\right)^{\delta / 2} \sum_{b=1}^{b_{a}} \sum_{u=1}^{u_{t}} E\left|\sum_{c=1}^{2^{b-1}} \sum_{v=1}^{2^{u-1}} W_{a b c}^{t u v}\right|^{\delta^{\prime}}\right.
$$




$$
\begin{aligned}
& +b_{a}^{\delta / 2} \sum_{b=1}^{b_{a}} E\left|\sum_{c=1}^{2^{b-1}} \sum_{v=1}^{2^{u_{t}-1}} W_{a b c}^{t\left(u_{t}+1\right)(2 v-1)}\right|^{\delta^{\prime}}+b_{a}^{\delta / 2} \sum_{b=1}^{b_{a}} E\left|\sum_{c=1}^{2^{b-1}} \sum_{v=1}^{2^{u_{t}-1}} W_{a b c}^{t\left(u_{t}+1\right)(2 v)}\right|^{\delta^{\prime}} \\
& +\left.u_{t}^{\delta / 2} \sum_{u=1}^{u_{t}} E\left|\sum_{c=1}^{2^{b_{a}-1}} \sum_{v=1}^{2^{u-1}} W_{a\left(b_{a}+1\right)(2 c-1)}^{t u v}+u_{t}^{\delta / 2} \sum_{u=1}^{u_{t}} E\right| \sum_{c=1}^{2^{b_{a}-1}} \sum_{v=1}^{2^{u-1}} W_{a\left(b_{a}+1\right)(2 c)}^{t u v}\right|^{\delta^{\prime}} \\
& +E\left|\sum_{c=1}^{2^{b_{a}-1}} \sum_{v=1}^{2^{u_{t}-1}} W_{a\left(b_{a}+1\right)(2 c-1)}^{t\left(u_{t}+1\right)(2 v-1)}\right|^{\delta^{\prime}}+E\left|\sum_{c=1}^{2^{b_{a}-1}} \sum_{v=1}^{2^{u_{t}-1}} W_{a\left(b_{a}+1\right)(2 c)}^{t\left(u_{t}+1\right)(2 v-1)}\right|^{\delta^{\prime}} \\
& \left.+E\left|\sum_{c=1}^{2^{b_{a}-1}} \sum_{v=1}^{2^{u_{t}-1}} W_{a\left(b_{a}+1\right)(2 c-1)}^{t\left(u_{t}+1\right)(2 v)}\right|^{\delta^{\prime}}+E\left|\sum_{c=1}^{2^{b_{a}-1}} \sum_{v=1}^{2^{u_{t}-1}} W_{a\left(b_{a}+1\right)(2 c)}^{t\left(u_{t}+1\right)(2 v)}\right|^{\delta^{\prime}}\right)
\end{aligned}
$$

By the construction of the sets $\mathcal{I}_{1 a b c}^{(1)}$ and $\mathcal{I}_{1 \text { tuv }}^{(2)}$, all double sums within absolute values above are double sums of independent random variables. Therefore, by Inequality $\mathrm{B}$, with $\lambda=\delta^{\prime}$,

$$
\begin{aligned}
& E\left|W_{\mathbf{1}}\right|^{\delta^{\prime}} \leq 2^{1+\delta} 3^{\delta} \sum_{a, t=1}^{2}\left(\left(b_{a} u_{t}\right)^{\delta / 2} \sum_{b=1}^{b_{a}} \sum_{u=1}^{u_{t}} \sum_{c=1}^{2^{b^{-1}}} \sum_{v=1}^{2^{u-1}} E\left|W_{a b c}^{t u v}\right|^{\delta^{\prime}}\right. \\
& +b_{a}^{\delta / 2} \sum_{b=1}^{b_{a}} \sum_{c=1}^{2^{b-1}} \sum_{v=1}^{2^{u_{t}-1}} E\left|W_{a b c}^{t\left(u_{t}+1\right)(2 v-1)}\right|^{\delta^{\prime}}+b_{a}^{\delta / 2} \sum_{b=1}^{b_{a}} \sum_{c=1}^{2^{b-1}} \sum_{v=1}^{2^{u_{t}-1}} E \mid W_{a b c}^{t\left(u_{t}+1\right)(2 v)||^{\delta^{\prime}}} \\
& +u_{t}^{\delta / 2} \sum_{u=1}^{u_{t}} \sum_{c=1}^{2^{b_{a}-1}} \sum_{v=1}^{2^{u-1}} E\left|W_{a\left(b_{a}+1\right)(2 c-1)}^{t u v}\right|^{\delta^{\prime}}+u_{t}^{\delta / 2} \sum_{u=1}^{u_{t}} \sum_{c=1}^{2^{b_{a}-1}} \sum_{v=1}^{2^{u-1}} E\left|W_{a\left(b_{a}+1\right)(2 c)}^{t u v}\right|^{\delta^{\prime}} \\
& +\left.\sum_{c=1}^{2^{b_{a}-1}} \sum_{v=1}^{2^{u_{t}-1}} E\left|W_{a\left(b_{a}+1\right)(2 c-1)}^{\left.t\left(u_{t}+1\right)(2 v-1)\right|^{\delta^{\prime}}}+\sum_{c=1}^{2^{b_{a}-1}} \sum_{v=1}^{2^{u_{t}-1}} E\right| W_{a\left(b_{a}+1\right)(2 c)}^{t\left(u_{t}+1\right)(2 v-1)}\right|^{\delta^{\prime}} \\
& +\sum_{c=1}^{2^{b_{a}-1}} \sum_{v=1}^{2^{u_{t}-1}} E\left|W_{a\left(b_{a}+1\right)(2 c-1)}^{t\left(u_{t}+1\right)(2 v)}\right|^{\delta^{\prime}}+\sum_{c=1}^{2^{b_{a}-1}} \sum_{v=1}^{2^{u_{t}-1}} E\left|W_{a\left(b_{a}+1\right)(2 c)}^{t\left(u_{t}+1\right)(2 v)}\right|
\end{aligned}
$$

Below, $\sum_{i_{1}}$ is the sum over all $i_{1}$ such that $\left(i_{1}, l_{1}\right) \in \mathcal{I}_{1 a b c}^{(1)}$, and $\sum_{l_{1}}$ is the sum over all $l_{1}$ such that $\left(i_{1}, l_{1}\right) \in \mathcal{I}_{1 a b c}^{(1)}$. In the same way, $\sum_{i_{2}}$ is the sum over all $i_{2}$ such that $\left(i_{2}, l_{2}\right) \in \mathcal{I}_{1 \text { tuv }}^{(2)}$, and $\sum_{l_{2}}$ is the sum over all $l_{2}$ such that $\left(i_{2}, l_{2}\right) \in \mathcal{I}_{1 t u v}^{(2)}$. For all pairs of "rectangles" $\mathcal{I}_{1 a b c}^{(1)}$ and $\mathcal{I}_{1 \text { tuv }}^{(2)}$, we can rewrite $W_{a b c}^{t u v}$ in the following way,

$$
\begin{aligned}
& W_{a b c}^{t u v}=\frac{1}{N}\left(\sum_{i_{1}} \sum_{i_{2}} X_{i} \sum_{l_{1}} \sum_{l_{2}} X_{\boldsymbol{l}}\right. \\
& +\sum_{i_{1}} \sum_{i_{2}} X_{i} \sum_{l_{1}} \sum_{l_{2}} w_{l_{1}}^{\prime} w_{l_{2}}^{\prime} X_{\boldsymbol{l}}+\sum_{i_{1}} \sum_{i_{2}} w_{i_{1}}^{\prime} w_{i_{2}}^{\prime} X_{i} \sum_{l_{1}} \sum_{l_{2}} X_{\boldsymbol{l}}
\end{aligned}
$$




$$
\begin{aligned}
& +\sum_{i_{1}} \sum_{i_{2}} X_{i} \sum_{l_{1}} \sum_{l_{2}} w_{l_{1}}^{\prime} X_{\boldsymbol{l}}+\sum_{i_{1}} \sum_{i_{2}} X_{i} \sum_{l_{1}} \sum_{l_{2}} w_{l_{2}}^{\prime} X_{\boldsymbol{l}} \\
& -\sum_{i_{1}} \sum_{i_{2}} w_{i_{1}}^{\prime} X_{i} \sum_{l_{1}} \sum_{l_{2}} X_{\boldsymbol{l}}-\sum_{i_{1}} \sum_{i_{2}} w_{i_{2}}^{\prime} X_{i} \sum_{l_{1}} \sum_{l_{2}} X_{\boldsymbol{l}} \\
& \left.-\sum_{i_{1}} \sum_{i_{2}} w_{i_{1}}^{\prime} X_{i} \sum_{l_{1}} \sum_{l_{2}} w_{l_{2}}^{\prime} X_{\boldsymbol{l}}-\sum_{i_{1}} \sum_{i_{2}} w_{i_{2}}^{\prime} X_{i} \sum_{l_{1}} \sum_{l_{2}} w_{l_{1}}^{\prime} X_{\boldsymbol{l}}\right),
\end{aligned}
$$

where $w_{i_{j}}^{\prime}=\left(k_{j}-i_{j}\right) / k_{j}$ and $w_{l_{j}}^{\prime}=\left(k_{j}-l_{j}\right) / k_{j}, j=1,2$. Note, on the right hand side of (18) we have $\left|w_{i_{j}}^{\prime}\right| \leq 1$ and $\left|w_{l_{j}}^{\prime}\right| \leq 1$, for all $i_{j}$ and $l_{j}, j=1,2$. Therefore, by Inequality A with $\lambda=\delta^{\prime}$ and the Cauchy-Schwarz inequality, it follows that $E\left|W_{a b c}^{t u v}\right|^{\delta^{\prime}}$ is bounded from above by $3^{\delta} / N^{\delta^{\prime}}$ times a sum of nine terms, all of the type

$$
\left(E\left|\sum_{i_{1}} \sum_{i_{2}} \alpha_{i} X_{i}\right|^{2 \delta^{\prime}} E\left|\sum_{l_{1}} \sum_{l_{2}} \beta_{\boldsymbol{l}} X_{\boldsymbol{l}}\right|^{2 \delta^{\prime}}\right)^{1 / 2}
$$

where $\left|\alpha_{i}\right| \leq 1$ and $\left|\beta_{\boldsymbol{l}}\right| \leq 1$ for all $\boldsymbol{i}$ and $\boldsymbol{l}$. Each side of $\mathcal{I}_{1 a b c}^{(1)}$ is less than or equal to $k_{1} / 2^{b}+1$, and each side of $\mathcal{I}_{1 \text { tuv }}^{(2)}$ is less than or equal to $k_{2} / 2^{u}+1$. Thus, if both $\mathcal{I}_{1 a b c}^{(1)}$ and $\mathcal{I}_{1 \text { tuv }}^{(2)}$ have rectangular shape, then by Lemma $1(i i)$,

$$
E\left|W_{a b c}^{t u v}\right|^{\delta^{\prime}} \leq \tau_{\delta} \eta\left(\frac{576 M}{N}\left(\frac{k_{1}}{2^{b}}+1\right)\left(\frac{k_{2}}{2^{u}}+1\right)\right)^{\delta^{\prime}} \leq L_{1}\left(\frac{K}{N 2^{b+u}}\right)^{\delta^{\prime}},
$$

where $L_{1}=\tau_{\delta} \eta(2304 M)^{\delta^{\prime}}$.

If $\mathcal{I}_{1 a b c}^{(1)}$ and $\mathcal{I}_{1 \text { tuv }}^{(2)}$ are two "triangular" sets, then since the number of points in these sets are not more than $\left(2 m_{1}+1\right)^{2}$ and $\left(2 m_{2}+1\right)^{2}$, respectively, we get by applying Inequality $\mathrm{A}$, with $\lambda=\delta^{\prime}$ and $r \leq\left(2 m_{1}+1\right)^{2}\left(2 m_{2}+1\right)^{2} \leq 81 M^{2}$,

$$
\begin{aligned}
E\left|W_{a b c}^{t u v}\right|^{\delta^{\prime}} \leq & \frac{(9 M)^{\delta}}{N^{\delta^{\prime}}} \sum_{\left(i_{1}, l_{1}\right) \in \mathcal{I}_{1 a b c}^{(1)}\left(i_{2}, l_{2}\right) \in \mathcal{I}_{1 t u v}^{(2)}} E\left|X_{i} X_{l}\right|^{\delta^{\prime}} \\
\leq & \frac{(9 M)^{\delta}}{N^{\delta^{\prime}}} \sum_{\left(i_{1}, l_{1}\right) \in \mathcal{I}_{1 a b c}^{(1)}} \sum_{\left.i_{2}, l_{2}\right) \in \mathcal{I}_{1 t u v}^{(2)}}\left(E\left|X_{i}\right|^{2 \delta^{\prime}} E\left|X_{l}\right|^{2 \delta^{\prime}}\right)^{1 / 2} \leq \frac{L_{2}}{N^{\delta^{\prime}}}
\end{aligned}
$$

where $L_{2}=\tau_{\delta}(9 M)^{2 \delta^{\prime}}$. Similarly, if $\mathcal{I}_{1 a b c}^{(1)}$ is "triangular" and $\mathcal{I}_{1 \text { tuv }}^{(2)}$ "rectangular",

$$
E\left|W_{a b c}^{t u v}\right|^{\delta^{\prime}} \leq L_{3}\left(\frac{\sqrt{K}}{N 2^{u}}\right)^{\delta^{\prime}},
$$

where $L_{3}=\tau_{\delta} \eta 108^{2 \delta^{\prime}} M^{3 / 2+3 \delta / 4}$. If $\mathcal{I}_{1 a b c}^{(1)}$ is "rectangular" and $\mathcal{I}_{1 t u v}^{(2)}$ "triangular",

$$
E\left|W_{a b c}^{t u v}\right|^{\delta^{\prime}} \leq L_{3}\left(\frac{\sqrt{K}}{N 2^{b}}\right)^{\delta^{\prime}}
$$


Although the sets $\mathcal{I}_{1111}^{(1)}$ and $\mathcal{I}_{1111}^{(2)}$ do not have a rectangular shape, inequality (20) holds in these cases as well. This is easily seen, e.g. we can "replace" $\mathcal{I}_{1111}^{(1)}$ with the "rectangular" set $\widetilde{\mathcal{I}}_{1111}^{(1)}$, defined as $\mathcal{I}_{1111}^{(1)}$ but without the requirement $\left(i_{1}, l_{1}\right) \in \mathcal{I}_{11}^{(1)}$ in the definition, and by (temporarily) defining all $X_{\boldsymbol{i}}$ and $X_{\boldsymbol{l}}$ with $\left(i_{1}, l_{1}\right) \in \widetilde{\mathcal{I}}_{1111}^{(1)} \backslash \mathcal{I}_{1111}^{(1)}$ as zero. Thus, all sums over the set $\mathcal{I}_{1111}^{(1)}$ will be equal to those over the "rectangular" set $\widetilde{\mathcal{I}}_{1111}^{(1)}$, and we can proceed as above.

Now we use inequalities (20)-(23) to proceed the estimation of (17). We get,

$$
\begin{aligned}
& E\left|W_{\mathbf{1}}\right|^{\delta^{\prime} \leq} \leq 2^{1+\delta} 3^{\delta} \sum_{a, t=1}^{2}\left(\left(b_{a} u_{t}\right)^{\delta / 2} \sum_{b=1}^{b_{a}} \sum_{u=1}^{u_{t}} \sum_{c=1}^{2^{b-1}} \sum_{v=1}^{2^{u-1}} L_{1}\left(\frac{K}{N 2^{b+u}}\right)^{\delta^{\prime}}\right. \\
&+2 b_{a}^{\delta / 2} \sum_{b=1}^{b_{a}} \sum_{c=1}^{2^{b-1}} \sum_{v=1}^{2^{u_{t}-1}} L_{3}\left(\frac{\sqrt{K}}{N 2^{b}}\right)^{\delta^{\prime}} \\
&\left.+2 u_{t}^{\delta / 2} \sum_{u=1}^{u_{t}} \sum_{c=1}^{2^{b_{a}-1}} \sum_{v=1}^{2^{u-1}} L_{3}\left(\frac{\sqrt{K}}{N 2^{u}}\right)^{\delta^{\prime}}+4 \sum_{c=1}^{2^{b_{a}-1}} \sum_{v=1}^{2^{u_{t}-1}} \frac{L_{2}}{N^{\delta^{\prime}}}\right) \\
& \leq 2^{1+\delta} 3^{\delta} \sum_{a, t=1}^{2}\left(\frac{L_{1}\left(b_{a} u_{t}\right)^{\delta / 2}}{4\left(1-(1 / 2)^{\delta / 2}\right)^{2}}\left(\frac{K}{N}\right)^{\delta^{\prime}}\right. \\
&+L_{3} \frac{\left.b_{a}^{\delta / 2} 2^{u_{t}-1}+u_{t}^{\delta / 2} 2^{b_{a}-1}\left(\frac{\sqrt{K}}{N}\right)^{\delta^{\prime}}+\frac{L_{2} 2^{b_{a}+u_{t}}}{N^{\delta^{\prime}}}\right)^{1-(1 / 2)^{\delta / 2}}}{\leq} \\
& 2^{3+} 3^{\delta}\left(\frac{L_{1}\left(\log _{2}\left(2 k_{1}\right) \log _{2}\left(2 k_{2}\right)\right)^{\delta / 2}}{4\left(1-(1 / 2)^{\delta / 2}\right)^{2}}\left(\frac{K}{N}\right)^{\delta^{\prime}}\right. \\
&\left.+L_{3} \frac{\left(\log _{2}\left(2 k_{1}\right)\right)^{\delta / 2} k_{2}+\left(\log _{2}\left(2 k_{2}\right)\right)^{\delta / 2} k_{1}}{1-(1 / 2)^{\delta / 2}}\left(\frac{\sqrt{K}}{N}\right)^{\delta^{\prime}}+\frac{4 L_{2} K}{N^{\delta^{\prime}}}\right)
\end{aligned}
$$

where the last inequality follows from the inequalities $b_{a} \leq \log _{2}\left(2 k_{1}\right), a=1,2$, and $u_{t} \leq \log _{2}\left(2 k_{2}\right), u=1,2$ (of which (15) is a special case).

Inequality (24) is valid for $W_{j}$ also when $j_{1}, j_{2}>1$, which can be shown by using the methods applied above. By definition, $\sum_{g_{1}, g_{2}=1}^{3}\left|\mathcal{E}_{\boldsymbol{g}}\right| \leq\left(n_{1} / k_{1}+\right.$ $1)\left(n_{2} / k_{2}+1\right) \leq 4 N / K$. Thus, from (14) and (24), it follows that

$$
\begin{aligned}
E\left|Q_{n}^{(4 b)}\right|^{\delta^{\prime}} & \leq 3^{2 \delta} 2^{6+\delta}\left(\frac{L_{1}\left(\log _{2}\left(2 k_{1}\right) \log _{2}\left(2 k_{2}\right)\right)^{\delta / 2}}{4\left(1-(1 / 2)^{\delta / 2}\right)^{2}}\left(\frac{K}{N}\right)^{\delta / 2}\right. \\
& \left.+L_{3} \frac{\left(\log _{2}\left(2 k_{1}\right)\right)^{\delta / 2} k_{2}+\left(\log _{2}\left(2 k_{2}\right)\right)^{\delta / 2} k_{1}}{1-(1 / 2)^{\delta / 2}} \frac{1}{\sqrt{K}}\left(\frac{\sqrt{K}}{N}\right)^{\delta / 2}+\frac{4 L_{2}}{N^{\delta / 2}}\right) .
\end{aligned}
$$

By the assumptions on $k_{1}=k_{1}\left(n_{1}\right)$ and $k_{2}=k_{2}\left(n_{2}\right)$ in the case when $0<\delta<2$, 
the right hand side above tends to zero as $n_{1}, n_{2} \rightarrow \infty$, and thus, by the Chebyshev inequality, $Q_{n}^{(4 b)} \stackrel{P}{\longrightarrow} 0$ as $n_{1}, n_{2} \rightarrow \infty$.

When $\delta=2$, a more direct approach can be used. In this case,

$$
E W_{j}^{2}=\sum_{\left(i_{1}, l_{1}\right) \in \mathcal{I}_{j_{1}}^{(1)}} \sum_{\left(i_{2}, l_{2}\right) \in \mathcal{I}_{j_{2}}^{(2)}} \sum_{\left(i_{1}^{\prime}, l_{1}^{\prime}\right) \in \mathcal{I}_{j_{1}}^{(1)}} \sum_{\left(i_{2}^{\prime}, l_{2}^{\prime}\right) \in \mathcal{I}_{j_{2}}^{(2)}} \frac{\alpha_{\boldsymbol{i} \boldsymbol{i}^{\prime}} \boldsymbol{l l}^{\prime} E X_{\boldsymbol{i}} X_{\boldsymbol{l}} X_{\boldsymbol{i}^{\prime}} X_{\boldsymbol{l}^{\prime}}}{N^{2}},
$$

where the non-random coefficients $\alpha_{i i^{\prime}} l \boldsymbol{l}^{\prime} \in[0,1]$. Note that the expected value of $X_{\boldsymbol{i}} X_{\boldsymbol{l}} X_{\boldsymbol{i}^{\prime}} X_{\boldsymbol{l}^{\prime}}$ is zero if $i_{1}+m_{1}<i_{1}^{\prime}, i_{1}^{\prime}+m_{1}<i_{1}, l_{1}+m_{1}<l_{1}^{\prime}$, or $l_{1}^{\prime}+m_{1}<l_{1}$, since in these cases at least one of the random variables is independent of the others. Also, the expected value is zero if $m_{2}+\max \left\{i_{2}, l_{2}^{\prime}\right\}<l_{2}, m_{2}+\max \left\{i_{2}^{\prime}, l_{2}\right\}<l_{2}^{\prime}$, $m_{2}+i_{2}<\min \left\{l_{2}, i_{2}^{\prime}\right\}$, or $m_{2}+i_{2}^{\prime}<\min \left\{i_{2}, l_{2}^{\prime}\right\}$. Thus, the number of non-zero terms in (41) is not more than $6\left(2 m_{1}+1\right)^{2}\left(2 m_{2}+1\right)^{2} K^{2} \leq 486 M^{2} K^{2}$. When $\delta=2$,

$$
E\left|X_{i} X_{\boldsymbol{l}} X_{\boldsymbol{i}^{\prime}} X_{\boldsymbol{l}^{\prime}}\right| \leq E X_{i}^{4}+E X_{\boldsymbol{l}}^{4}+E X_{\boldsymbol{i}^{\prime}}^{4}+E X_{\boldsymbol{l}^{\prime}}^{4} \leq 4 \tau_{\delta}
$$

which implies that $E W_{j}^{2} \leq 1944 \tau_{\delta} M^{2} K^{2} / N^{2}$. Recall that $\sum_{g_{1}, g_{2}=1}^{3}\left|\mathcal{E}_{\boldsymbol{g}}\right| \leq$ $4 N / K$. By inequality (14), with $\delta=2, E\left|Q_{n}^{(4 b)}\right|^{2} \leq 139968 \tau_{\delta} M^{2} K / N \rightarrow 0$, as $n_{1}, n_{2} \rightarrow \infty$, and therefore $Q_{n}^{(4 b)} \stackrel{P}{\longrightarrow} 0$ as $n_{1}, n_{2} \rightarrow \infty$. By similar arguments as above we see that $Q_{n}^{(4 a)}, Q_{n}^{(4 c)}, Q_{n}^{(4 d)}$, and $Q_{n}^{(4 e)}$, all tend to zero in probability as $n_{1}, n_{2} \rightarrow \infty$. Hence,

$$
Q_{n}^{(4)} \stackrel{P}{\longrightarrow} 0, \text { as } n_{1}, n_{2} \rightarrow \infty .
$$

By (7), and the Cauchy-Schwarz and the Lyapunov inequalities, respectively, one can verify that

$$
\gamma_{\boldsymbol{n}} \leq \tau_{\delta}^{2 /\left(2 \delta^{\prime}\right)}\left(1+2\left(m_{1}+m_{2}\right)+4 M\right), \text { for all } n_{1}, n_{2} .
$$

Thus, we see that

$$
E\left|Q_{n}^{(5)}\right| \leq \frac{4 K b_{n} \gamma_{n}}{N^{2}} \rightarrow 0, \text { as } n_{1}, n_{2} \rightarrow \infty
$$

By the Cauchy-Schwarz inequality, Inequality A, and Lemma 1(ii), respectively,

$$
\begin{aligned}
& E\left|Q_{n}^{(6)}\right|^{\delta^{\prime}} \leq\left(\frac{E|X . .|^{2 \delta^{\prime}}}{N^{4+2 \delta}} E\left|\sum_{h_{1}=1-k_{1}}^{k_{1}-1} \sum_{h_{2}=1-k_{2}}^{k_{2}-1} \sum_{i_{1}=1-0 \wedge h_{1}}^{n_{1}-0 \vee h_{1}} \sum_{i_{2}=1-0 \wedge h_{2}}^{n_{2}-0 \vee h_{2}} X_{\boldsymbol{i}} w_{h_{1}} w_{h_{2}}\right|^{2 \delta^{\prime}}\right)^{1 / 2} \\
& \leq\left(\frac{E|X . .|^{2 \delta^{\prime}}(4 K)^{1+\delta}}{N^{4+2 \delta}} \sum_{h_{1}=1-k_{1}}^{k_{1}-1} \sum_{h_{2}=1-k_{2}}^{k_{2}-1} E\left|\sum_{i_{1}=1-0 \wedge h_{1}}^{n_{1}-0 \vee h_{1}} \sum_{i_{2}=1-0 \wedge h_{2}}^{n_{2}-0 \vee h_{2}} X_{i}\right|^{2 \delta^{\prime}}\right)^{1 / 2} \\
& \leq \tau_{\delta} \eta\left(\frac{256 M K}{N}\right)^{\delta^{\prime}} \rightarrow 0, \text { as } n_{1}, n_{2} \rightarrow \infty .
\end{aligned}
$$


From (27) and (28) we see that

$$
Q_{n}^{(5)} \stackrel{P}{\longrightarrow} 0 \text { and } Q_{n}^{(6)} \stackrel{P}{\longrightarrow} 0, \text { as } n_{1}, n_{2} \rightarrow \infty,
$$

which, together with the results (11)-(13) and (26), implies that

$$
\hat{\gamma}_{\boldsymbol{n}}-\gamma_{\boldsymbol{n}} \stackrel{P}{\longrightarrow} 0, \text { as } n_{1}, n_{2} \rightarrow \infty \text {. }
$$

Proof of Theorem 2. Throughout the proof (Lemma 2 and 3 included) we will, without loss of generality, assume that $n_{i}>k_{i}>m_{i} \geq 1, i=1,2$. Define $\delta^{\prime}=\delta^{\prime}$, $X_{i}=Y_{\boldsymbol{i}}-\mu_{\boldsymbol{i}}, \boldsymbol{i} \in \mathcal{A}_{\boldsymbol{n}}, \tilde{\mu}=\hat{\mu}-\mu=X . . / N, \tilde{r}_{i_{2}}=\hat{r}_{i_{2}}-r_{i_{2}}=X{ }_{i_{2}} / n_{1}-\tilde{\mu}, i_{2}=1, \ldots, n_{2}$, and $\tilde{c}_{i_{1}}=\hat{c}_{i_{1}}-c_{i_{1}}=X_{i_{1}} \cdot / n_{2}-\tilde{\mu}, i_{1}=1, \ldots, n_{1}$. Then

$$
\begin{aligned}
\hat{\gamma}_{\boldsymbol{n}}^{\left(n e^{\prime}\right)}-\gamma_{\boldsymbol{n}} & =\frac{1}{K N} \sum_{i \in \mathcal{A}_{n}^{\prime}}\left(\sum_{j \in B_{i}}\left(X_{j}-\tilde{\mu}-\tilde{r}_{j_{2}}-\tilde{c}_{j_{1}}\right) I_{j}\right)^{2}-\frac{E X_{*}^{2}}{N} \\
= & \frac{1}{K N} \sum_{i \in \mathcal{A}_{n}^{\prime}}\left(\sum_{j \in B_{i}} X_{j} I_{j}\right)^{2}-\frac{E X_{*}^{2}}{N} \\
& +\frac{1}{K N} \sum_{i \in \mathcal{A}_{n}^{\prime}}\left(\sum_{j \in B_{i}}\left(\tilde{\mu}+\tilde{r}_{j_{2}}+\tilde{c}_{j_{1}}\right) I_{j}\right)^{2} \\
& -\frac{2}{K N} \sum_{i \in \mathcal{A}_{n}^{\prime}}\left(\sum_{j \in B_{i}} X_{j} I_{j}\right)\left(\sum_{j \in B_{i}}\left(\tilde{\mu}+\tilde{r}_{j_{2}}+\tilde{c}_{j_{1}}\right) I_{j}\right) .
\end{aligned}
$$

From the the proof of Theorem 2, we have

$$
\begin{aligned}
& \frac{1}{K N} \sum_{\boldsymbol{i} \in \mathcal{A}_{n}^{\prime}}\left(\sum_{j \in B_{\boldsymbol{i}}} X_{\boldsymbol{j}} I_{\boldsymbol{j}}\right)^{2}-\frac{E X^{2} .}{N} \\
& =Q_{\boldsymbol{n}}^{(1)}+2 Q_{\boldsymbol{n}}^{(2)}+2 Q_{\boldsymbol{n}}^{(3)}+2 Q_{n}^{(4)} \stackrel{P}{\longrightarrow} 0, \text { as } n_{1}, n_{2} \rightarrow \infty .
\end{aligned}
$$

Let $w_{h_{i}}=\left(k_{i}-\left|h_{i}\right|\right) / k_{i}, i=1,2$. We need to show that

$$
\begin{aligned}
& Q_{\boldsymbol{n}}^{(7)}=\frac{1}{K N} \sum_{\boldsymbol{i} \in \mathcal{A}_{n}^{\prime}}\left(\sum_{\boldsymbol{j} \in B_{i}}\left(\tilde{\mu}+\tilde{r}_{j_{2}}+\tilde{c}_{j_{1}}\right) I_{j}\right)^{2} \\
& =\frac{1}{N} \sum_{h_{1}=1-k_{1}}^{k_{1}-1} \sum_{h_{2}=1-k_{2}}^{k_{2}-1} \sum_{i_{1}=1-0 \wedge h_{1}}^{n_{1}-0 \vee h_{1}} \sum_{i_{2}=1-0 \wedge h_{2}}^{n_{2}-0 \vee h_{2}}\left(\tilde{\mu}+\tilde{r}_{i_{2}}+\tilde{c}_{i_{1}}\right)\left(\tilde{\mu}+\tilde{r}_{i_{2}+h_{2}}+\tilde{c}_{i_{1}+h_{1}}\right) w_{h_{1}} w_{h_{2}}
\end{aligned}
$$

tends to zero in probability as $n_{1}, n_{2} \rightarrow \infty$. By symmetry arguments, it is enough to show that

$$
\frac{1}{N} \sum_{i \in \mathcal{A}_{n}}\left(\tilde{\mu}+\tilde{r}_{i_{2}}+\tilde{c}_{i_{1}}\right)\left(\tilde{\mu}+\tilde{r}_{i_{2}}+\tilde{c}_{i_{1}}\right)=\tilde{\mu}^{2}+\frac{1}{n_{2}} \sum_{i_{2}=1}^{n_{2}} \tilde{r}_{i_{2}}^{2}+\frac{1}{n_{1}} \sum_{i_{1}=1}^{n_{1}} \tilde{c}_{i_{1}}^{2},
$$




$$
\begin{aligned}
& \frac{1}{N} \sum_{h_{2}=1}^{k_{2}-1} \sum_{i_{1}=1}^{n_{1}} \sum_{i_{2}=1}^{n_{2}-h_{2}}\left(\tilde{\mu}+\tilde{r}_{i_{2}}+\tilde{c}_{i_{1}}\right)\left(\tilde{\mu}+\tilde{r}_{i_{2}+h_{2}}+\tilde{c}_{i_{1}}\right) w_{h_{1}} w_{h_{2}}, \\
& \frac{1}{N} \sum_{h_{1}=1}^{k_{1}-1} \sum_{h_{2}=1}^{k_{2}-1} \sum_{i_{1}=1}^{n_{1}-h_{1}} \sum_{i_{2}=1}^{n_{2}-h_{2}}\left(\tilde{\mu}+\tilde{r}_{i_{2}}+\tilde{c}_{i_{1}}\right)\left(\tilde{\mu}+\tilde{r}_{i_{2}+h_{2}}+\tilde{c}_{i_{1}+h_{1}}\right) w_{h_{1}} w_{h_{2}},
\end{aligned}
$$

all tend to zero in probability. By Lemma $1(i i)$,

$$
E|\tilde{\mu}|^{2 \delta^{\prime}}=\frac{E|X . .|^{2 \delta^{\prime}}}{N^{2 \delta^{\prime}}} \leq \frac{\tau_{\delta} \eta(64 M)^{\delta^{\prime}}}{N^{\delta^{\prime}}} .
$$

Let

$$
\begin{aligned}
& G_{\boldsymbol{n}}^{(1)}=\frac{1}{n_{1}} \sum_{h_{1}=1}^{m_{1}} \sum_{i_{1}=1}^{n_{1}-h_{1}} w_{h_{1}} \tilde{c}_{i_{1}} \tilde{c}_{i_{1}+h_{1}}, \quad G_{\boldsymbol{n}}^{(2)}=\frac{1}{n_{1}} \sum_{h_{1}=m_{1}+1}^{k_{1}-1} \sum_{i_{1}=1}^{n_{1}-h_{1}} w_{h_{1}} \tilde{c}_{i_{1}} \tilde{c}_{i_{1}+h_{1}} \\
& H_{n}^{(1)}=\frac{1}{n_{2}} \sum_{h_{2}=1}^{m_{2}} \sum_{i_{2}=1}^{n_{2}-h_{2}} w_{h_{2}} \tilde{r}_{i_{2}} \tilde{r}_{i_{2}+h_{2}}, \quad H_{n}^{(2)}=\frac{1}{n_{2}} \sum_{h_{2}=m_{2}+1}^{k_{2}-1} \sum_{i_{2}=1}^{n_{2}-h_{2}} w_{h_{2}} \tilde{r}_{i_{2}} \tilde{r}_{i_{2}+h_{2}} .
\end{aligned}
$$

Lemma 2 Assume that $\mathrm{AM}, \operatorname{AD}(\boldsymbol{m})$, and $\mathrm{AL}(\delta)$ are valid. Then,

$$
E\left|G_{\boldsymbol{n}}^{(1)}\right|^{\delta^{\prime}} \leq S_{1} / n_{2}^{\delta^{\prime}}, \quad E\left|H_{\boldsymbol{n}}^{(1)}\right|^{\delta^{\prime}} \leq S_{2} / n_{1}^{\delta^{\prime}},
$$

where $S_{i}=\tau_{\delta} \eta\left(256 M m_{i}\right)^{\delta^{\prime}}, i=1,2$. If $0<\delta<2$, then

$$
E\left|G_{n}^{(2)}\right|^{\delta^{\prime}} \leq \frac{T_{1}\left(k_{1} \log _{2}\left(2 k_{1}\right)\right)^{\delta / 2}+T_{1}^{\prime}}{n_{2} N^{\delta / 2}}, \quad E\left|H_{n}^{(2)}\right|^{\delta^{\prime}} \leq \frac{T_{2}\left(k_{2} \log _{2}\left(2 k_{2}\right)\right)^{\delta / 2}+T_{2}^{\prime}}{n_{1} N^{\delta / 2}},
$$

where $T_{i}=3^{\delta} \tau_{\delta}(2 \eta)^{2}\left(92160 m_{i} M\right)^{\delta^{\prime}}\left(1-(1 / 2)^{\delta / 2}\right)^{-1}$ and $T_{i}^{\prime}=3^{\delta} 2^{4} \tau_{\delta} \eta\left(4608 m_{i}^{2} M\right)^{\delta^{\prime}}$, $i=1,2$. If $\delta=2$, then

$$
E\left|G_{\boldsymbol{n}}^{(2)}\right|^{\delta^{\prime}} \leq T_{1}^{\prime \prime} k_{1} /\left(n_{2} N\right), \quad E\left|H_{\boldsymbol{n}}^{(2)}\right|^{\delta^{\prime}} \leq T_{2}^{\prime \prime} k_{2} /\left(n_{1} N\right),
$$

where $T_{i}^{\prime \prime}=2^{20} 3^{3} \tau_{\delta} \eta m_{i}^{2} M^{2}, i=1,2$.

Proof. We only prove the inequalities for $G_{n}^{(i)}, i=1,2$. By Inequality A and the Cauchy-Schwarz inequality, respectively,

$$
E\left|G_{\boldsymbol{n}}^{(1)}\right|^{\delta^{\prime}} \leq \frac{m_{1}^{\delta / 2}}{n_{1}} \sum_{h_{1}=1}^{m_{1}} \sum_{i_{1}=1}^{n_{1}-h_{1}}\left(E\left|\tilde{c}_{i_{1}}\right|^{2 \delta^{\prime}} E\left|\tilde{c}_{i_{1}+h_{1}}\right|^{2 \delta^{\prime}}\right)^{1 / 2} .
$$

By Inequality $\mathrm{A}$ and Lemma 1 we have, for $i_{1}=1, \ldots, n_{1}$,

$$
\begin{aligned}
E\left|\tilde{c}_{i_{1}}\right|^{2 \delta^{\prime}} & =E\left|\frac{X_{i_{1}} \cdot}{n_{2}}-\tilde{\mu}\right|^{2 \delta^{\prime}} \leq 2^{1+\delta}\left(E\left|\frac{X_{i_{1}}}{n_{2}}\right|^{2 \delta^{\prime}}+E|\tilde{\mu}|^{2 \delta^{\prime}}\right) \\
& \leq 2^{1+\delta}\left(\frac{\tau_{\delta} \eta\left(8 m_{2}\right)^{\delta^{\prime}}}{n_{2}^{\delta^{\prime}}}+\frac{\tau_{\delta} \eta(64 M)^{\delta^{\prime}}}{N^{\delta^{\prime}}}\right) \leq \frac{\tau_{\delta} \eta(256 M)^{\delta^{\prime}}}{n_{2}^{\delta^{\prime}}}
\end{aligned}
$$


which, together with (35), completes the proof of the inequality for $G_{\boldsymbol{n}}^{(1)}$.

For the proof of the inequality for $G_{\boldsymbol{n}}^{(2)}$ in the case when $0<\delta<2$, the approach (and notation) used for $Q_{n}^{(4 b)}$ in the proof of Theorem 2 will be used. Let $\mathcal{K}_{j_{1}}=\left\{j_{1}: \boldsymbol{j} \in \mathcal{K}_{\boldsymbol{j}}\right\}$ and $\mathcal{E}_{g_{1}}=\left\{g_{1}: \boldsymbol{g}=\left(g_{1}, g_{2}\right) \in \mathcal{E}_{\boldsymbol{g}}\right\}$. Then, if we define

$$
W_{j_{1}}=\sum_{\left(i_{1}, l_{1}\right) \in \mathcal{I}_{j_{1}}^{(1)}} \frac{k_{1}-\left(l_{1}-i_{1}\right)}{k_{1} n_{1}} \tilde{c}_{i_{1}} \tilde{c}_{l_{1}}
$$

we can write $G_{\boldsymbol{n}}^{(2)}=\sum_{g_{1}=1}^{3} \sum_{j_{1} \in \mathcal{E}_{g_{1}}} W_{j_{1}}$. By Inequality A with $\lambda=\delta^{\prime}$ and $r=3$, and Inequality B with $\lambda=\delta^{\prime}$ and $r=\left|\mathcal{E}_{g_{1}}\right|$,

$$
E\left|G_{n}^{(2)}\right|^{\delta^{\prime}} \leq 3^{\delta / 2} 2 \sum_{g_{1}=1}^{3} \sum_{j_{1} \in \mathcal{E}_{g_{1}}} E\left|W_{j_{1}}\right|^{\delta^{\prime}}
$$

All the $E\left|W_{j_{1}}\right|^{\delta^{\prime}}$ can be handled similarly, and therefore only the case $j_{1}=1$ will be considered below.

Define

$$
W_{a b c}=W_{1 a b c}=\sum_{\left(i_{1}, l_{1}\right) \in \mathcal{I}_{1 a b c}^{(1)}} \frac{k_{1}-\left(l_{1}-i_{1}\right)}{k_{1} n_{1}} \tilde{c}_{i_{1}} \tilde{c}_{l_{1}} .
$$

Then

$$
W_{1}=\sum_{a=1}^{2}\left(\sum_{b=1}^{b_{a}} \sum_{c=1}^{2^{b-1}} W_{a b c}+\sum_{c=1}^{2^{b_{a}-1}} W_{a\left(b_{a}+1\right)(2 c-1)}+\sum_{c=1}^{2^{b_{a}-1}} W_{a\left(b_{a}+1\right)(2 c)}\right) .
$$

By going through steps like (16)-(18), and with

$$
\left(E\left|\sum_{i_{1}} \alpha_{i_{1}} \tilde{c}_{i_{1}}\right|^{2 \delta^{\prime}} E\left|\sum_{l_{1}} \beta_{l_{1}} \tilde{c}_{l_{1}}\right|^{2 \delta^{\prime}}\right)^{1 / 2}, \quad\left|\alpha_{i_{1}}\right| \leq 1 \text { and }\left|\beta_{l_{1}}\right| \leq 1 \text { for all } i_{1}, l_{1} \text {, }
$$

(which can be handled like (36)), instead of (19), we get

$$
E\left|W_{a b c}\right|^{\delta^{\prime}} \leq L_{1}^{\prime}\left(\frac{k_{1}}{N 2^{b}}\right)^{\delta^{\prime}}
$$

where $L_{1}^{\prime}=\tau_{\delta} \eta^{2}\left(46080 m_{1} M\right)^{\delta^{\prime}}$, for the case when $\mathcal{I}_{1 a b c}^{(1)}$ have a rectangular shape.

If $\mathcal{I}_{1 a b c}^{(1)}$ is "triangular", then the number of points in this set is not more than $\left(2 m_{1}+1\right)^{2}$. Thus, by Inequality A, with $\lambda=\delta^{\prime}$ and $r \leq\left(2 m_{1}+1\right)^{2} \leq 9 m_{1}^{2}$, 
and inequality (36), respectively,

$$
\begin{aligned}
E\left|W_{a b c}\right|^{\delta^{\prime}} & \leq \frac{\left(3 m_{1}\right)^{\delta}}{n_{1}^{\delta^{\prime}}} \sum_{\left(i_{1}, l_{1}\right) \in \mathcal{I}_{1 a b c}^{(1)}} E\left|\tilde{c}_{i_{1}} \tilde{c}_{l_{1}}\right|^{\delta^{\prime}} \\
& \leq \frac{\left(3 m_{1}\right)^{\delta}}{n_{1}^{\delta^{\prime}}} \sum_{\left(i_{1}, l_{1}\right) \in \mathcal{I}_{1 a b c}^{(1)}}\left(E\left|\tilde{c}_{i_{1}}\right|^{2 \delta^{\prime}} E\left|\tilde{c}_{l_{1}}\right|^{2 \delta^{\prime}}\right)^{1 / 2} \leq \frac{L_{2}^{\prime}}{N^{\delta^{\prime}}},
\end{aligned}
$$

where $L_{2}^{\prime}=\tau_{\delta} \eta\left(2304 M m_{1}^{2}\right)^{\delta^{\prime}}$.

Now we use inequalities (38) and (39) to proceed the estimation of (17). We get,

$$
\begin{aligned}
E\left|W_{1}\right|^{\delta^{\prime}} & \leq 2^{\delta^{\prime}} 3^{\delta / 2} \sum_{a=1}^{2}\left(b_{a}^{\delta / 2} \sum_{b=1}^{b_{a}} \sum_{c=1}^{2^{b-1}} L_{1}^{\prime}\left(\frac{k_{1}}{N 2^{b}}\right)^{\delta^{\prime}}+2 \sum_{c=1}^{2^{b_{a}-1}} \frac{L_{2}^{\prime}}{N^{\delta^{\prime}}}\right) \\
& \leq 2^{\delta^{\prime}} 3^{\delta / 2} \sum_{a=1}^{2}\left(\frac{L_{1}^{\prime} b_{a}^{\delta / 2}}{2\left(1-(1 / 2)^{\delta / 2}\right)}\left(\frac{k_{1}}{N}\right)^{\delta^{\prime}}+\frac{L_{2}^{\prime} 2^{b_{a}}}{N^{\delta^{\prime}}}\right) \\
& \leq 2^{2 \delta^{\prime} / 2} 3^{\delta / 2}\left(\frac{L_{1}^{\prime}\left(\log _{2}\left(2 k_{1}\right)\right)^{\delta / 2}}{2\left(1-(1 / 2)^{\delta / 2}\right)}\left(\frac{k_{1}}{N}\right)^{\delta^{\prime}}+\frac{2 L_{2}^{\prime} k_{1}}{N^{\delta^{\prime}}}\right)
\end{aligned}
$$

where the last inequality follows from the inequality $b_{a} \leq \log _{2}\left(2 k_{1}\right), a=1,2$ (of which (15) is a special case).

Inequality (40) is valid for $W_{j_{1}}$ also when $j_{1}>1$, which can be shown by using the methods applied above. By definition, $\sum_{g_{1}=1}^{3}\left|\mathcal{E}_{g_{1}}\right| \leq n_{1} / k_{1}+1 \leq$ $2 n_{1} / k_{1}$. Thus, from (37) and (40), it follows that

$$
E\left|G_{n}^{(2)}\right|^{\delta^{\prime}} \leq \frac{3^{\delta} 2^{5+\delta / 2}}{n_{2} N^{\delta / 2}}\left(\frac{L_{1}^{\prime}\left(k_{1} \log _{2}\left(2 k_{1}\right)\right)^{\delta / 2}}{4\left(1-(1 / 2)^{\delta / 2}\right)}+L_{2}^{\prime}\right)
$$

which completes the proof for $G_{\boldsymbol{n}}^{(2)}$ in the case when $0<\delta<2$.

Next, assume $\delta=2$. In this case,

$$
E W_{j_{1}}^{2}=\sum_{\left(i_{1}, l_{1}\right) \in \mathcal{I}_{j_{1}}^{(1)}} \sum_{\left(i_{1}^{\prime}, l_{1}^{\prime}\right) \in \mathcal{I}_{j_{1}}^{(1)}} \frac{\alpha_{i_{1} i_{1}^{\prime} l_{1} l_{1}^{\prime}} E \tilde{c}_{i_{1}} \tilde{c}_{l_{1}} \tilde{c}_{i_{1}^{\prime}} \tilde{c}_{l_{1}^{\prime}}}{n_{1}^{2}}
$$

where the non-random coefficients $\alpha_{i_{1} i_{1}^{\prime} l_{1} l_{1}^{\prime}} \in[0,1]$. The expected value of $\tilde{c}_{i_{1}} \tilde{c}_{l_{1}} \tilde{c}_{i_{1}^{\prime}} \tilde{l}_{l_{1}^{\prime}}$ is zero if $i_{1}+m_{1}<i_{1}^{\prime}, i_{1}^{\prime}+m_{1}<i_{1}, l_{1}+m_{1}<l_{1}^{\prime}$, or $l_{1}^{\prime}+m_{1}<l_{1}$, since in these cases at least one of the random variables is independent of the others. Thus, the number of non-zero terms in (41) is not more than $\left(2 m_{1}+1\right)^{2} k_{1}^{2} \leq\left(3 m_{1} k_{1}\right)^{2}$. By (36), with $\delta=2$,

$$
E\left|\tilde{c}_{i_{1}} \tilde{l}_{l_{1}} \tilde{c}_{i_{1}^{\prime}} \tilde{c}_{l_{1}^{\prime}}\right| \leq E \tilde{c}_{i_{1}}^{4}+E \tilde{c}_{l_{1}}^{4}+E \tilde{c}_{i_{1}^{\prime}}^{4}+E \tilde{c}_{l_{1}^{\prime}}^{4} \leq 2^{18} \tau_{\delta} \eta\left(M / n_{2}\right)^{2},
$$


which implies that $E W_{j_{1}}^{2} \leq 2^{18} \tau_{\delta} \eta\left(3 m_{1} k_{1} M / N\right)^{2}$. Recall that $\sum_{g_{1}=1}^{3}\left|\mathcal{E}_{g_{1}}\right| \leq$ $2 n_{1} / k_{1}$. Thus, by inequality (37) and with $\delta=2$, we obtain $E\left|G_{n}^{(2)}\right|^{\delta^{\prime}} \leq$ $2^{20} 3^{3} \tau_{\delta} \eta m_{1}^{2} M^{2} k_{1} /\left(n_{2} N\right)$.

Define

$$
\begin{array}{ll}
G_{\boldsymbol{n}}^{(3)}=\frac{1}{n_{1}} \sum_{h_{1}=1}^{k_{1}-1} \sum_{i_{1}=1}^{n_{1}-h_{1}} \tilde{c}_{i_{1}} w_{h_{1}}, & G_{\boldsymbol{n}}^{(4)}=\frac{1}{n_{1}} \sum_{h_{1}=1}^{k_{1}-1} \sum_{i_{1}=1}^{n_{1}-h_{1}} \tilde{c}_{i_{1}+h_{1}} w_{h_{1}}, \\
H_{\boldsymbol{n}}^{(3)}=\frac{1}{n_{2}} \sum_{h_{2}=1}^{k_{2}-1} \sum_{i_{2}=1}^{n_{2}-h_{2}} \tilde{r}_{i_{2}} w_{h_{2}}, & H_{\boldsymbol{n}}^{(4)}=\frac{1}{n_{2}} \sum_{h_{2}=1}^{k_{2}-1} \sum_{i_{2}=1}^{n_{2}-h_{2}} \tilde{r}_{i_{2}+h_{2}} w_{h_{2}} .
\end{array}
$$

Lemma 3 If $\mathrm{AM}, \operatorname{AD}(\boldsymbol{m})$, and $\mathrm{AL}(\delta)$ are valid, then

$$
\begin{aligned}
& E\left|G_{\boldsymbol{n}}^{(i)}\right|^{2 \delta^{\prime}} \leq \tau_{\delta} \eta^{2}\left(2048 m_{1} M\right)^{\delta^{\prime}}\left(\frac{k_{1}^{3}}{n_{1} N}\right)^{\delta^{\prime}}, \quad i=3,4, \\
& E\left|H_{\boldsymbol{n}}^{(i)}\right|^{2 \delta^{\prime}} \leq \tau_{\delta} \eta^{2}\left(2048 m_{2} M\right)^{\delta^{\prime}}\left(\frac{k_{2}^{3}}{n_{2} N}\right)^{\delta^{\prime}}, \quad i=3,4 .
\end{aligned}
$$

Proof. Here we only give a proof for $H_{n}^{(4)}$. Since $\sum_{i_{2}=1}^{n_{2}} \tilde{r}_{i_{2}}=0$, we can write

$$
H_{n}^{(4)}=-\frac{1}{n_{2}} \sum_{h_{2}=1}^{k_{2}-1} \sum_{i_{2}=1}^{h_{2}} w_{h_{2}} \tilde{r}_{i_{2}}=-\frac{1}{n_{2}} \sum_{i_{2}=1}^{k_{2}-1} \tilde{r}_{i_{2}} \sum_{h_{2}=i_{2}}^{k_{2}-1} w_{h_{2}} \text {, }
$$

where the last equality follows by changing the order of summation. Let $\alpha_{i_{2}}=$ $k_{2}^{-1} \sum_{h_{2}=i_{2}}^{k_{2}-1} w_{h_{2}}$, and note that $\left|\alpha_{i_{2}}\right| \leq 1, i_{2}=1, \ldots, k_{2}-1$. By Lemma $1(i)$, with $\tilde{r}_{i_{2}}$ instead of $X_{i}$, and with the bound

$$
E\left|\tilde{r}_{i_{2}}\right|^{2 \delta^{\prime}} \leq \tau_{\delta} \eta\left(256 M / n_{1}\right)^{\delta^{\prime}}
$$

(derived as inequality (36)) instead of the bound $E\left|X_{\boldsymbol{i}}\right|^{2 \delta^{\prime}}<\tau_{\delta}$, we get

$$
E\left|\frac{k_{2}}{n_{2}} \sum_{i_{2}=1}^{k_{2}-1} \alpha_{i_{2}} \tilde{r}_{i_{2}}\right|^{2 \delta^{\prime}} \leq \tau_{\delta} \eta^{2}\left(2048 m_{2} M\right)^{\delta^{\prime}}\left(\frac{k_{2}^{3}}{n_{2} N}\right)^{\delta^{\prime}}
$$

which was to be proved.

Now, we return to the sums (31)-(33). From (34), (36), and (42), it is easily seen that the sum (31) converges to zero in probability. That the sums (32) and (33) converges to zero in probability can be verified from Lemma 2 and 3, together with inequality (34) (for the first of these two sums, inequality (36) is also needed). 
What remains to be shown is that also

$$
\begin{aligned}
Q_{n}^{(8)} & =\frac{-2}{K N} \sum_{i \in \mathcal{A}_{n}^{\prime}}\left(\sum_{j \in B_{i}} X_{j} I_{j}\right)\left(\sum_{j \in B_{i}}\left(\tilde{\mu}+\tilde{r}_{j_{2}}+\tilde{c}_{j_{1}}\right) I_{j}\right) \\
& =-\frac{2}{N} \sum_{h_{1}=1-k_{1}}^{k_{1}-1} \sum_{h_{2}=1-k_{2}}^{k_{2}-1} \sum_{i_{1}=1-0 \wedge h_{1}}^{n_{1}-0 \vee h_{1}} \sum_{i_{2}=1-0 \wedge h_{2}}^{n_{2}-0 \vee h_{2}} X_{\boldsymbol{i}}\left(\tilde{\mu}+\tilde{r}_{i_{2}+h_{2}}+\tilde{c}_{i_{1}+h_{1}}\right) w_{h_{1}} w_{h_{2}}
\end{aligned}
$$

converges to zero in probability as $n_{1}, n_{2} \rightarrow \infty$. By symmetry arguments, it is enough to show that

$$
\frac{1}{N} \sum_{i \in \mathcal{A}_{n}} X_{\boldsymbol{i}}\left(\tilde{\mu}+\tilde{r}_{i_{2}}+\tilde{c}_{i_{1}}\right), \quad \frac{1}{N} \sum_{h_{2}=1}^{k_{2}-1} \sum_{i_{1}=1}^{n_{1}-h_{1}} \sum_{i_{2}=1}^{n_{2}-h_{2}} X_{\boldsymbol{i}}\left(\tilde{\mu}+\tilde{r}_{i_{2}+h_{2}}+\tilde{c}_{i_{1}}\right) w_{h_{1}} w_{h_{2}},
$$

and

$$
\frac{1}{N} \sum_{h_{1}=1}^{k_{1}-1} \sum_{h_{2}=1}^{k_{2}-1} \sum_{i_{1}=1}^{n_{1}-h_{1}} \sum_{i_{2}=1}^{n_{2}-h_{2}} X_{\boldsymbol{i}}\left(\tilde{\mu}+\tilde{r}_{i_{2}+h_{2}}+\tilde{c}_{i_{1}+h_{1}}\right) w_{h_{1}} w_{h_{2}}
$$

all tend to zero in probability. The first of these three sums is actually equal to sum (31), which has been shown to tend to zero in probability. The proofs for the last two are similar, and therefore we give a proof only for the last, more difficult, one.

By the Cauchy-Schwarz inequality, Inequality A, and Lemma 1(ii), respectively, it follows as in (28) that

$$
E\left|\frac{\tilde{\mu}}{N} \sum_{h_{1}=1}^{k_{1}-1} \sum_{h_{2}=1}^{k_{2}-1} \sum_{i_{1}=1}^{n_{1}-h_{1}} \sum_{i_{2}=1}^{n_{2}-h_{2}} X_{\boldsymbol{i}} w_{h_{1}} w_{h_{2}}\right|^{\delta^{\prime}} \leq \tau_{\delta} \eta\left(\frac{64 K M}{N}\right)^{\delta^{\prime}} .
$$

Let $\tilde{\tilde{r}}_{i_{2} h_{1}}=n_{1}^{-1} \sum_{i_{1}=1}^{n_{1}-h_{1}} X_{\boldsymbol{i}}, h_{1}=1, \ldots, k_{1}-1$, and $\tilde{\tilde{c}}_{i_{1} h_{2}}=n_{2}^{-1} \sum_{i_{2}=1}^{n_{2}-h_{2}} X_{\boldsymbol{i}}, h_{2}=$ $1, \ldots, k_{2}-1$, and define

$$
\begin{array}{ll}
\tilde{G}_{\boldsymbol{n}}^{(1)}=\frac{1}{n_{1}} \sum_{h_{1}=1}^{m_{1}} \sum_{i_{1}=1}^{n_{1}-h_{1}} w_{h_{1}} \tilde{\tilde{c}}_{i_{1} h_{2}} \tilde{c}_{i_{1}+h_{1}}, & \tilde{G}_{\boldsymbol{n}}^{(2)}=\frac{1}{n_{1}} \sum_{h_{1}=m_{1}+1}^{k_{1}-1} \sum_{i_{1}=1}^{n_{1}-h_{1}} w_{h_{1}} \tilde{\tilde{c}}_{i_{1} h_{2}} \tilde{c}_{i_{1}+h_{1}}, \\
\tilde{H}_{\boldsymbol{n}}^{(1)}=\frac{1}{n_{2}} \sum_{h_{2}=1}^{m_{2}} \sum_{i_{2}=1}^{n_{2}-h_{2}} w_{h_{2}} \tilde{\tilde{r}}_{i_{2} h_{1}} \tilde{r}_{i_{2}+h_{2}}, & \tilde{H}_{\boldsymbol{n}}^{(2)}=\frac{1}{n_{2}} \sum_{h_{2}=m_{2}+1}^{k_{2}-1} \sum_{i_{2}=1}^{n_{2}-h_{2}} w_{h_{2}} \tilde{\tilde{r}}_{i_{2} h_{1}} \tilde{r}_{i_{2}+h_{2}} .
\end{array}
$$

The upper bounds for $E\left|\tilde{c}_{i_{1}}\right|^{2 \delta^{\prime}}$ and $E\left|\tilde{r}_{i_{2}}\right|^{2 \delta^{\prime}}$, given in (36) and (42), are upper bounds for $E\left|\tilde{\tilde{c}}_{i_{1} h_{2}}\right|^{2 \delta^{\prime}}$ and $E\left|\tilde{\tilde{r}}_{i_{2} h_{1}}\right|^{2 \delta^{\prime}}$, respectively, as well. Thus, it is not too difficult to see that the results given for $G_{n}^{(i)}$ and $H_{n}^{(i)}$ in Lemma 2, are valid also for $\tilde{G}_{n}^{(i)}$ and $\tilde{H}_{n}^{(i)}, i=1,2$, respectively. From this and (44) it follows that 
the sum (43) tends to zero in probability. Thus, we have shown that both $Q_{n}^{(7)}$ and $Q_{n}^{(7)}$ tend to zero in probability, and this, together with (29) and (30), completes the proof of Theorem 2 .

Proof of Corollary 1. By (8) and Inequality A, $E\left(\hat{\gamma}_{\boldsymbol{n}}^{(n e)}-\gamma_{\boldsymbol{n}}\right)^{2} \leq 24 \sum_{i=1}^{6} E\left(Q_{n}^{(i)}\right)^{2}$. In the proof of Theorem 1 it was shown that $E\left(Q_{n}^{(2)}\right)^{2}=O\left(N^{-1}\right)$ and $E\left(Q_{n}^{(i)}\right)^{2}=$ $O\left(K N^{-1}\right), i=4,6$. That $E\left(Q_{n}^{(i)}\right)^{2}=O\left(N^{-1}\right), i=1,5$, follows by applying the same approach as for $i=2$. Finally, by Inequality A, $E\left(Q_{n}^{(3)}\right)^{2}=O\left(k_{1}^{-2}+k_{2}^{-2}\right)$.

Proof of Corollary 2. From the proof of Theorem 2, $\hat{\gamma}_{\boldsymbol{n}}^{\left(n e^{\prime}\right)}-\gamma_{\boldsymbol{n}}^{\prime}=Q_{\boldsymbol{n}}^{(1)}+$ $2 \sum_{i=2}^{4} Q_{n}^{(i)}+\sum_{i=7}^{8} Q_{n}^{(i)}$, and therefore, by Inequality A, $E\left(\hat{\gamma}_{\boldsymbol{n}}^{\left(n e^{\prime}\right)}-\gamma_{\boldsymbol{n}}^{\prime}\right)^{2} \leq$ $24\left(\sum_{i=1}^{4} E\left(Q_{n}^{(i)}\right)^{2}+\sum_{i=7}^{8} E\left(Q_{n}^{(i)}\right)^{2}\right)$. From Lemma 2 and 3, together with (34), (36), and (42), it follows that $E\left(Q_{n}^{(i)}\right)^{2}=O\left(K N^{-1}+k_{1}^{2} n_{1}^{-2}+k_{2}^{2} n_{2}^{-2}\right), i=7,8$ (for $i=8$, note that the results given for $G_{n}^{(i)}$ and $H_{n}^{(i)}$ in Lemma 2, are valid also for $\tilde{G}_{n}^{(i)}$ and $\tilde{H}_{n}^{(i)}, i=1,2$, respectively). For $E\left(Q_{n}^{(i)}\right)^{2}, i=1,2,3,4$, see the proof of Corollary 1.

\section{ACKNOWLEDGEMENTS}

This work is a part of the Swedish research programme Remote Sensing for the Environment, RESE (home page http://rese.satellus.se), partly financed by the Swedish Fundation for Strategic Environmental Research, MISTRA.

\section{BIBLIOGRAPHY}

Belyaev, Yu. K. (1995). Bootstrap, resampling and Mallows metric. Lecture Notes 1, Department of Mathematical Statistics, Umeå University, Sweden.

Belyaev, Yu. K. (1996). Central limit resampling theorems for $m$-dependent heterogeneous random variables. Research Report 1996-5, Department of Mathematical Statistics, Umeå University, Sweden.

Belyaev, Yu. K. and Sjöstedt, S. (1996). Resampling theorems for vector valued heterogeneous random variables. Research Report 1996-9, Department of Mathematical Statistics, Umeå University, Sweden.

Belyaev, Yu. K. and Sjöstedt-de Luna, S. (2000). "Weakly approaching sequences of random distributions," J. Appl. Probab., 37, 807-822.

Cressie, N. (1993). Statistics for spatial data, 2nd edn. Wiley, New York. 
Mercer, W. B. and Hall, A. D. (1911). "The experimental error of field trials," J. Agric. Sci., 4, 107-132.

Petrov, V. V. (1995). Limit theorems of probability theory: sequences of independent random variables. Oxford University Press, Oxford.

Politis, D. N. (1993). "Nonparametric resampling for homogeneous strong mixing random fields," J. Multivariate Anal., 47, 301-328.

Politis, D. N., Romano, J. P., and Wolf, M. (1999). Subsampling. Springer, New York.

Possolo, A. (1991). "Subsampling a random field," Spatial statistics and imaging (Ed. A. Possolo), 286-294. IMS Lecture notes 20, Hayward, CA.

Sherman, M. (1996). "Variance estimation for statistics computed from spatial lattice data," J. Roy. Statist. Soc. Ser. B, 58, 509-523.

Sherman, M. and Carlstein, E. (1994). "Nonparametric estimation of the moments of a general statistic computed from spatial data," J. Amer. Statist. Assoc., 89, 496-500. 
Serien Arbetsrapporter utges i första hand för institutionens eget behov av viss dokumentation. Rapporterna är indelade i följande grupper: Riksskogstaxeringen, Planering och inventering, Biometri, Fjärranalys, Kompendier och undervisningsmaterial, Examensarbeten samt internationellt. Författarna svarar själva för rapporternas vetenskapliga innehåll.

This series of Working Papers reflects the activity of this Department of Forest Resource Management and Geomatics. The sole responsibility for the scientific content of each Working Paper relies on the respective author.

\section{Riksskogstaxeringen: (The Swedish National Forest Inventory)}

19951 Kempe, G. Hjälpmedel för bestämning av slutenhet i plant- och ungskog. ISRN SLU-SRG-AR--1--SE

2 Riksskogstaxeringen och Ståndortskarteringen vid regional miljöövervakning. - metoder för att förbättra upplösningen vid inventering i skogliga avrinningsområden. ISRN SLU-SRG-AR--2--SE.

199723 Lundström, A., Nilsson, P. \& Ståhl, G. Certifieringens konsekvenser för möjliga uttag av industri- och energived. - En pilotstudie. ISRN SLU-SRG-AR--23--SE.

24 Fridman, J. \& Walheim, M. Död ved i Sverige. - Statistik från Riksskogstaxeringen. ISRN SLU-SRG-AR--24--SE.

199830 Fridman, J. \& Kihlblom, D. \& Söderberg, U. Förslag till miljöindexsystem för naturtypen skog. ISRN SLU-SRG-AR--30--SE.

34 Löfgren, P. Skogsmark, samt träd- och buskmark inom fjällområdet. En skattning av arealer enligt internationella ägoslagsdefinitioner. ISRN SLU-SRG-AR--34--SE.

37 Odell, G. \& Ståhl, G. Vegetationsförändringar i svensk skogsmark mellan 1980- och 90-talet. -En studie grundad på Ståndortskarteringen. ISRN SLU-SRG-AR--37--SE.

38 Lind, T. Quantifying the area of edge zones in Swedish forest to assess the impact of nature conservation on timber yields. ISRN SLU-SRG-AR--38--SE.

199950 Ståhl, G., Walheim, M. \& Löfgren, P. Fjällinventering. - En utredning av innehåll och design. ISRN SLU-SRG--AR--50--SE.

52 Riksskogstaxeringen inför 2000-talet. - Utredningar avseende innehåll och omfattning i en framtida Riksskogstaxering. Redaktörer: Jonas Fridman \& Göran Ståhl.

ISRN SLU-SRG-AR--52--SE.

54 Fridman, J. m.fl. Sveriges skogsmarksarealer enligt internationella ägoslagsdefinitioner. ISRN SLU-SRG-AR--54--SE.

56 Nilsson, P. \& Gustafsson, K. Skogsskötseln vid 90-talets mitt - läge och trender. ISRN SLU-SRG-AR--56--SE. 
57 Nilsson, P. \& Söderberg, U. Trender i svensk skogsskötsel - en intervjuundersökning. ISRN SLU-SRG-AR--57--SE.

199961 Broman, N \& Christoffersson, J. Mätfel i provträdsvariabler och dess inverkan på precision och noggrannhet i volymskattningar. ISRN SLU-SRG-AR--61--SE.

65 Hallsby, G m.fl. Metodik för skattning av lokala skogsbränsleresurser.

ISRN SLU-SRG-AR--65--SE.

75 von Segebaden, G. Komplement till "RIKSTAXEN 75 ÅR". ISRN SLU-SRG-AR--75--SE.

200186 Lind, T. Kolinnehåll i skog och mark i Sverige - Baserat på Riksskogstaxeringens data. ISRN SLU-SRG-AR--86--SE

\section{Planering och inventering: (Forest inventory and planning)}

19953 Holmgren, P. \& Thuresson, T. Skoglig planering på amerikanska västkusten - intryck från en studieresa till Oregon, Washington och British Columbia 1-14 augusti 1995. ISRN SLU-SRG-AR--3--SE.

4 Ståhl, G. The Transect Relascope - An Instrument for the Quantification of Coarse Woody Debris. ISRN SLU-SRG-AR--4--SE

199615 van Kerkvoorde, M. A sequential approach in mathematical programming to include spatial aspects of biodiversity in long range forest management planning.

ISRN SLU-SRG-AR--15--SE.

199718 Christoffersson, P. \& Jonsson, P. Avdelningsfri inventering - tillvägagångssätt och tidsåtgång. ISRN SLU-SRG-AR--18--SE.

19 Ståhl, G., Ringvall, A. \& Lämås, T. Guided transect sampling - An outline of the principle. ISRN SLU-SRGL-AR--19--SE.

25 Lämås, T. \& Ståhl, G. Skattning av tillstånd och förändringar genom inventeringssimulering - En handledning till programpaketet "NVSIM".

ISRN SLU-SRG-AR--25--SE.

26 Lämås, T. \& Ståhl, G. Om dektering av förändringar av populationer i begränsade områden. ISRN SLU-SRG-AR--26--SE.

199959 Petersson, H. Biomassafunktioner för trädfraktioner av tall, gran och björk i Sverige. ISRN SLU-SRG-AR--59--SE.

63 Fridman, J., Löfstrand, R \& Roos, S. Stickprovsvis landskapsövervakning - En förstudie. ISRN SLU-SRG-AR--63--SE.

200068 Nyström, K. Funktioner för att skatta höjdtillväxten i ungskog.

ISRN SLU-SRG-AR--68--SE. 
70 Walheim, M. \& Löfgren, P. Metodutveckling för vegetationsövervakning i fjällen. ISRN SLU-SRG-AR--70--SE.

73 Holm, S. \& Lundström, A. Åtgärdsprioriteter. ISRN SLU-SRG-AR--73--SE.

76 Fridman, J. \& Ståhl, G. Funktioner för naturlig avgång i svensk skog. ISRN SLU-SRG-AR--76--SE.

200182 Holmström, H. Averaging Absolute GPS Positionings Made Underneath Different Forest Canopies - A Splendid Example of Bad Timing in Research.

ISRN SLU-SRG-AR--79--SE.

\section{Biometri: (Biometrics)}

199722 Ali, Abdul Aziz. Describing Tree Size Diversity. ISRN SLU-SEG-AR--22--SE.

199964 Berhe, L. Spatial continuity in tree diameter distribution.

ISRN SLU-SRG-AR--64--SE

200188 Ekström, M. Nonparametric Estimation of the Variance of Sample Means Based on Nonstationary Spatial Data. ISRN SLU-SRG-AR--88--SE.

\section{Fjärranalys: (Remote Sensing)}

199728 Hagner, O. Satellitfjärranalys för skogsföretag. ISRN SLU-SRG-AR--28--SE.

29 Hagner, O. Textur till flygbilder för skattning av beståndsegenskaper. ISRN SLU-SRG-AR--29--SE.

199832 Dahlberg, U., Bergstedt, J. \& Pettersson, A. Fältinstruktion för och erfarenheter från vegetationsinventering i Abisko, sommaren 1997. ISRN SLU-SRG-AR--32--SE.

43 Wallerman, J. Brattåkerinventeringen. ISRN SLU-SRG-AR--28--SE.

199951 Holmgren, J., Wallerman, J. \& Olsson, H. Plot - Level Stem Volume Estimation and Tree Species Discrimination with Casi Remote Sensing. ISRN SLU-SRG-AR--51--SE.

53 Reese, H. \& Nilsson, M. Using Landsat TM and NFI data to estimate wood volume, tree biomass and stand age in Dalarna. ISRN SLU-SRG-AR--53--SE.

200066 Löfstrand, R., Reese, H. \& Olsson, H. Remote Sensing aided Monitoring of NonTimber Forest Resources - A literature survey. ISRN SLU-SRG-AR--66--SE.

69 Tingelöf, U \& Nilsson, M.Kartering av hyggeskanter i pankromaötiska SPOT-bilder. ISRN SLU-SRG-AR--69--SE. 
79 Reese, H \& Nilsson, M. Wood volume estimations for Älvsbyn Kommun using SPOT satellite data and NFI plots. ISRN SLU-SRG-AR--79--SE.

\section{Kompendier och undervisningsmaterial: (Compendia and educational papers)}

199614 Holm, S. \& Thuresson, T. samt jägm.studenter kurs 92/96. En analys av skogstillståndet samt några alternativa avverkningsberäkningar för en del av Östads säteri. ISRN SLU-SRG-AR--14--SE.

21 Holm, S. \& Thuresson, T. samt jägm.studenter kurs 93/97. En analys av skogsstillståndet samt några alternativa avverkningsberäkningar för en stor del av Östads säteri. ISRN SLU-SRG-AR--21--SE.

199842 Holm, S. \& Lämås, T. samt jägm.studenter kurs 93/97. An analysis of the state of the forest and of some management alternatives for the Östad estate.

ISRN SLU-SRG-AR--42--SE

199958 Holm, S. samt studenter vid Sveriges lantbruksuniversitet i samband med kurs i strategisk och taktisk skoglig planering år 1998. En analys av skogsstillståndet samt några alternativa avverknings beräkningar för Östads säteri. ISRN SLU-SRG-AR--58--SE.

200187 Eriksson, O. (Ed.) Strategier för Östads säteri: Redovisning av planer framtagna under kursen Skoglig planering ur ett företagsperspektiv HT2000, SLU Umeå. ISRN SLU-SRG-AR--87--SE.

\section{Examensarbeten: (Theses by Swedish forestry students)}

19955 Törnquist, K. Ekologisk landskapsplanering i svenskt skogsbruk - hur började det?. Examensarbete $\mathrm{i}$ ämnet skogsuppskattning och skogsindelning. ISRN SLU-SRG-AR--5--SE.

19966 Persson, S. \& Segner, U. Aspekter kring datakvaliténs betydelse för den kortsiktiga planeringen. Examensarbete i ämnet skogsuppskattning och skogsindelning. ISRN SLU-SRG-AR--6--SE.

7 Henriksson, L. The thinning quotient - a relevant description of a thinning? Gallringskvot - en tillförlitlig beskrivning av en gallring? Examensarbete i ämnet skogsuppskattning och skogsindelning. ISRN SLU-SRG-AR--7--SE.

8 Ranvald, C. Sortimentsinriktad avverkning. Examensarbete i ämnet skogsuppskattning och skogsindelning. ISRN SLU-SRG-AR--8--SE.

9 Olofsson, C. Mångbruk i ett landskapsperspektiv - En fallstudie på MoDo Skog AB, Örnsköldsviks förvaltning. Examensarbete i ämnet skogsuppskattning och skogsindelning. ISRN SLU-SRG-AR--9--SE.

10 Andersson, $\mathrm{H}$. Taper curve functions and quality estimation for Common Oak (Quercus Robur L.) in Sweden. Examensarbete i ämnet skogsuppskattning och skogsindelning. ISRN SLU-SRG-AR--10--SE. 
11 Djurberg, H. Den skogliga informationens roll i ett kundanpassat virkesflöde. - En bakgrundsstudie samt simulering av inventeringsmetoders inverkan på noggrannhet $\mathrm{i}$ leveransprognoser till sågverk. Examensarbete i ämnet skogsuppskattning och skogsindelning. ISRN SLU-SRG-AR--11--SE.

12 Bredberg, J. Skattning av ålder och andra beståndsvariabler - en fallstudie baserad på MoDo:s indelningsrutiner. Examensarbete i ämnet skogsuppskattning och skogsindelning. ISRN SLU-SRG-AR--14--SE.

13 Gunnarsson, F. On the potential of Kriging for forest management planning. Examensarbete i ämnet skogsuppskattning och skogsindelning. ISRN SLU-SRG-AR--13--SE.

16 Tormalm, K. Implementering av FSC-certififering av mindre enskilda markägares skogsbruk. Examensarbete i ämnet skogsuppskattning och skogsindelning. ISRN SLU-SRG-AR--16--SE.

199717 Engberg, M. Naturvärden i skog lämnad vid slutavverkning. - En inventering av upp till 35 år gamla föryngringsytor på Sundsvalls arbetsomsåde, SCA. Examensarbete i ämnet skogsuppskattning och skogsindelning. ISRN-SLU-SRG-AR--17--SE.

20 Cedervind, J. GPS under krontak i skog. Examensarbete i ämnet skogsuppskattning och skogsindelning. ISRN SLU-SRG-AR--20--SE.

27 Karlsson, A. En studie av tre inventeringsmetoder i slutavverkningsbestånd. Examensarbete. ISRN SLU-SRG-AR--27--SE.

199831 Bendz, J. SÖDRAs gröna skogsbruksplaner. En uppföljning relaterad till SÖDRAs miljömål, FSC's kriterier och svensk skogspolitik. Examensarbete. ISRN SLU-SRG-AR--31--SE.

33 Jonsson, Ö. Trädskikt och ståndortsförhållanden i strandskog. - En studie av tre bäckar i Västerbotten. Examensarbete. ISRN SLU-SRG-AR--33--SE.

35 Claesson, S. Thinning response functions for single trees of Common oak (Quercus Robur L.) Examensarbete. ISRN SLU-SEG-AR--35--SE.

36 Lindskog, M. New legal minimum ages for final felling. Consequences and forest owner attitudes in the county of Västerbotten. Examensarbete.

ISRN SLU-SRG-AR--36--SE.

40 Persson, M. Skogsmarksindelningen i gröna och blå kartan - en utvärdering med hjälp av riksskogstaxeringens provytor. Examensarbete. ISRN SLU-SRG-AR--40--SE.

41 Eriksson, F. Markbaserade sensorer för insamling av skogliga data - en förstudie. Examensarbete. ISRN SLU-SRG-AR--41--SE.

45 Gessler, C. Impedimentens potientiella betydelse för biologisk mångfald. - En studie av myr- och bergimpediment i ett skogslandskap i Västerbotten. Examensarbete.

ISRN SLU-SRG-AR--45--SE. 
Gustafsson, K. Långsiktsplanering med geografiska hänsyn - en studie på Bräcke arbetsområde, SCA Forest and Timber. Examensarbete. ISRN SLU-SRG-AR--46--SE.

47 Holmgren, J. Estimating Wood Volume and Basal Area in Forest Compartments by Combining Satellite Image Data with Field Data. Examensarbete i ämnet Fjärranalys. ISRN SLU-SRG-AR--47--SE.

49 Härdelin, S. Framtida förekomst och rumslig fördelning av gammal skog. - En fallstudie på ett landskap i Bräcke arbetsområde. Examensarbete SCA. ISRN SLU-SRG-AR--49--SE.

199955 Imamovic, D. Simuleringsstudie av produktionskonsekvenser med olika miljömål. Examensarbete för Skogsstyrelsen. ISRN SLU-SRG-AR--55--SE

62 Fridh, L. Utbytesprognoser av rotstående skog. Examensarbete i skoglig planering. ISRN SLU-SRG-AR--62--SE.

200067 Jonsson, T. Differentiell GPS-mätning av punkter i skog. Point-accuracy for differential GPS under a forest canaopy. ISRN SLU-SRG-AR--67--SE.

71 Lundberg, N. Kalibrering av den multivariata variabeln trädslagsfördelning. Examensarbete i biometri. ISRN SLU-SRG-AR--71--SE.

72 Skoog, E. Leveransprecision och ledtid - två nyckeltal för styrning av virkesflödet. Examensarbete i skoglig planering. ISRN SLU-SRG-AR--72--SE.

74 Johansson, L. Rotröta i Sverige enligt Riksskogstaxeringen. Examens arbete i ämnet skogsindelning och skogsuppskattning. ISRN SLU-SRG-AR--74--SE.

77 Nordh, M. Modellstudie av potentialen för renbete anpassat till kommande slutavverkningar. Examensarbete på jägmästarprogrammet i ämnet skoglig planering. ISRN SLU-SRG-AR--77--SE.

78 Eriksson, D. Spatial Modeling of Nature Conservation Variables useful in Forestry Planning. Examensarbete. ISRN SLU-SRG-AR--74--SE.

81 Fredberg, K. Landskapsanalys med GIS och ett skogligt planeringssystem.

Examensarbete på skogsvetarprogrammet i ämnet skogshushållning. ISRN SLU-SRG-AR--81--SE.

83 Lindroos, O.Underlag för skogligt länsprogram Gotland. Examensarbete i ämnet skoglig planering. ISRN SLU-SRG-AR--83-SE.

84 Dahl, M. Satellitbildsbaserade skattningar av skogsområden med röjningsbehov. Examensarbete på akogsvetarprogrammet $\mathrm{i}$ ämnet skoglig planering. ISRN SLU-SRG-AR--84--SE.

85 Staland, J. Styrning av kundanpassade timmerflöden - Inverkan av traktbankens stlorlek och utbytesprognosens tillförlitlighet. Examensarbete i ämnet skoglig planering.

ISRN SLU-SRG-AR--85--SE. 
199839 Sandewall, Ohlsson, B \& Sandewall, R.K. People's options on forest land use - a research study of land use dynamics and socio-economic conditions in a historical perspective in the Upper Nam Nan Water Catchment Area, Lao PDR. ISRN SLU-SRG-AR--39--SE.

44 Sandewall, M., Ohlsson, B., Sandewall, R.K., Vo Chi Chung, Tran Thi Binh \& Pham Quoc Hung. People's options on forest land use. Government plans and farmers intentions - a strategic dilemma. ISRN SLU-SRG-AR--44--SE.

48 Sengthong, B. Estimating Growing Stock and Allowable Cut in LaoPDR using Data from Land Use Maps and the National Forest Inventory (NFI). Master thesis. ISRN SLU-SRG-AR--48--SE.

199960 Inter-active and dynamic approaches on forest and land-use planning - proceedings from a training workshop in Vietnam and Lao PDR, April 12-30, 1999. Edited by Mats Sandewall ISRN SLU-SRG-AR--60--SE.

200080 Sawathvong. S. Forest Land Use Planning in Nam Pui National Biodiversity Conservation Area, Lao P.D.R. ISRN SLU-SRG-AR--80--SE. 